\title{
Multisensory brain mechanisms of bodily self-consciousness
}

Olaf Blanke $1,2,3$

\section{Abstract | Recent research has linked bodily self-consciousness to the processing and} integration of multisensory bodily signals in temporoparietal, premotor, posterior parietal and extrastriate cortices. Studies in which subjects receive ambiguous multisensory information about the location and appearance of their own body have shown that these brain areas reflect the conscious experience of identifying with the body (self-identification (also known as body-ownership)), the experience of where 'l' am in space (self-location) and the experience of the position from where 'l' perceive the world (first-person perspective). Along with phenomena of altered states of self-consciousness in neurological patients and electrophysiological data from non-human primates, these findings may form the basis for a neurobiological model of bodily self-consciousness.

\section{Body ownership}

The feeling that the physical

body and its parts, such as its hands and feet, belong to 'me' and are 'my' body.
${ }^{1}$ Center for Neuroprosthetics, School of Life Sciences, École Polytechnique Fédérale de Lausanne, 1015 Lausanne, Switzerland.

${ }^{2}$ Laboratory of Cognitive Neuroscience, Brain Mind Institute, School of Life

Sciences, École Polytechnique Fédérale de Lausanne, 1015 Lausanne, Switzerland. ${ }^{3}$ Department of Neurology, University Hospital, 1211 Geneva, Switzerland. e-mail:olaf.blanke@epfl.ch doi:10.1038/nrn3292

Published online 18 July 2012
Human adults experience a 'real me' that 'resides' in 'my' body and is the subject (or 'I') of experience and thought. This aspect of self-consciousness, namely the feeling that conscious experiences are bound to the self and are experiences of a unitary entity ('I'), is often considered to be one of the most astonishing features of the human mind.

A powerful approach to investigate self-consciousness has been to target brain mechanisms that process bodily signals (that is, bodily self-consciousness) ${ }^{1-6}$. Experimentation with such bodily signals is complex as they are continuously present and updated and are conveyed by different senses, as well as through motor and visceral signals. However, recent developments in video, virtual reality and robotics technologies have allowed us to investigate the central mechanisms of bodily self-consciousness by providing subjects with ambiguous multisensory information about the location and appearance of their own body. This has made it possible to study three important aspects of bodily self-consciousness, how they relate to the processing of bodily signals and which functional and neural mechanisms they may share. These three aspects are: self-identification with the body (that is, the experience of owning a body), selflocation (that is, the experience of where I am in space) and the first-person perspective (that is, the experience from where I perceive the world).

This Review describes, for each of these aspects, the major experimental paradigms and behavioural findings, neuroimaging and neurological lesion data in humans, and electrophysiological studies in non-human primates, with the goal to develop a data-driven neurobiological model of bodily self-consciousness.

\section{Limb representation and self-consciousness}

Many of the recent approaches on bodily self-consciousness can be traced back to findings in patients with focal brain damage who had deficits in the processing of bodily signals $^{7-14}$. For example, 70 years ago, neurologist Josef Gerstmann ${ }^{15}$ described two patients with damage to the right temporoparietal cortex who experienced loss of ownership for their left arm and hand (ownership for the right extremities and the rest of their body was preserved). This condition is known as somatoparaphrenia ${ }^{9,15,16}$. Such patients most often selectively mis-attribute one of their limbs, mostly their contralesional hand, as belonging to another person. Another subset of patients with somatoparaphrenia may suffer from the opposite pattern and self-attribute the hands of other people, when these are presented in their contralesional hemispace, as belonging to themselves. Recent work has demonstrated that the intensity of somatoparaphrenia can be manipulated through various visual, somatosensory and cognitive procedures ${ }^{17,18}$, and that damage resulting in this condition centres on the right posterior insula ${ }^{19}$.

The rubber hand illusion. Research on body ownership was recently spurred by the observation that illusory ownership of a fake, dummy, rubber or virtual hand can be induced in healthy people $\mathrm{e}^{20-23}$. A seminal paper ${ }^{20}$ described a simple procedure that uses multisensory (in this case, visuotactile) conflicts to induce hand 
ownership for a rubber or fake hand: the rubber hand illusion. Viewing a fake hand being stroked by a paintbrush in synchrony with strokes applied to one's own corresponding (but occluded) hand can induce the illusion that the touch applied to the fake hand is felt and also induces illusory ownership for the fake hand (FIG. 1a). In addition, participants perceive their hand to be at a position that is displaced towards the fake hand's position - a phenomenon known as proprioceptive drift ${ }^{20,23,24}$. Illusory hand ownership is abolished or decreased when the visuotactile stroking is asynchronous $^{20}$, when an object (rather than a fake hand) is stroked ${ }^{23}$ or when the fake arm is not aligned with ${ }^{21,23}$ or is too distant from the participant's own $\operatorname{arm}^{25}$ (for reviews, see REFS 26,27).

Several conceptual models have proposed that illusory hand ownership is caused by visuo-proprioceptive integration that is further modulated by tactile stimulation $^{26-28}$. Although initial work suggested common brain mechanisms for illusory hand ownership and proprioceptive drift ${ }^{20}$, recent findings have suggested that distinct multisensory mechanisms underlie the two phenomena. In addition, they are modulated by different factors and rarely correlate in strength with each other ${ }^{24,28}$.

Brain areas and multimodal neurons involved in illusory limb ownership. Activation of the bilateral premotor cortex (PMC), regions in the intraparietal sulcus (IPS), insula and sensorimotor cortex have, in functional MRI (fMRI) and positron emission tomography (PET) studies, been associated with illusory limb ownership ${ }^{21,29-33}$ (FIG. 1 b). The cerebellum, insula, supplementary motor area, anterior cingulate cortex and posterior parietal cortex, as well as gamma oscillations over the sensorimotor cortex $^{31,32}$, have also been implicated ${ }^{21,29,33-35}$, whereas damage to pathways connecting the PMC, prefrontal cortex and parietal cortex results in an inability to experience illusory hand ownership ${ }^{36}$.

Makin and co-workers ${ }^{26}$ hypothesized that illusory hand ownership may involve trimodal neurons in the PMC and IPS that integrate tactile, visual and proprioceptive signals; such neurons have been described in non-human primates ${ }^{37-44}$. Indeed, PMC and IPS neurons often respond to stimuli applied to the skin of the contralateral $\mathrm{arm}^{40-42}$ and to visual stimuli approaching that hand or arm. Importantly, the visual receptive fields of these neurons are arm-centred, and their position in the visual field depends on proprioceptive signals: their spatial position shifts when the arm position is changed ${ }^{41-43}$ (FIG. 1C). It has been proposed that in the rubber hand illusion, merely seeing the fake hand or visuotactile stimulation of the fake hand and the occluded subject's hand may lead to a shift (or enlargement; see below) of the visual receptive fields of IPS and PMC neurons, so that they now also encode the position of the fake hand ${ }^{26}$. Such changes in receptive field properties have been shown to occur after tool and virtual reality hand use (FIG. 1c) in bimodal visuotactile IPS neurons (and probably in PMC neurons as well) in monkeys ${ }^{42,43}$ and are also compatible with data in humans ${ }^{45-47}$. Moreover, in monkeys, arm-centred trimodal IPS neurons can be induced to code for a seen fake arm after synchronous stroking of the fake arm and the (occluded) animal's own arm but not after asynchronous stroking ${ }^{48}$ (FIG. 1d).

\section{Body representation and self-consciousness}

The phenomena of somatoparaphrenia and the rubber hand illusion are important for studying limb ownership and perceived limb position. However, they do not enable us to investigate fundamental aspects of selfconsciousness that are related to the global and unitary character of the self. That is, the self is normally experienced as a single representation of the entire, spatially situated body rather than as a collection of several different body parts ${ }^{1}$. Indeed, patients with somatoparaphrenia and healthy subjects with illusory hand ownership still experience normal self-location, normal first-person perspective and normal self-identification with the rest of their body. These three crucial aspects of bodily self-consciousness also remain normal in many other interesting research paradigms and clinical conditions that alter ownership of fingers ${ }^{49,50}$, feet (in patients with somatoparaphrenia), half-bodies ${ }^{12,51,52}$ or faces ${ }^{53,54}$.

Investigations of patients suffering from a distinct group of neurological conditions have revealed that selfidentification, self-location and first-person perspective can be altered in so-called autoscopic phenomena ${ }^{51,55-57}$. These phenomena have directly inspired the development of experimental procedures - using video, virtual reality and/or robotic devices - that induce changes in self-location, self-identification and first-person perspective in healthy subjects ${ }^{58-60}$. The subjects experience illusions, referred to as out-of-body illusions or fullbody illusions, that arise from visuotactile and visuovestibular conflicts. In such studies, the tactile stroking stimulus is applied to the back or chest of a participant who is being filmed and simultaneously views (through a head-mounted display (HMD)) the stroking of a human body in a real-time film or virtual reality animation (FIG. 2).

Experimental approaches. One approach involved participants viewing a three-dimensional video image on an HMD that was linked to a video camera that was placed $2 \mathrm{~m}$ behind the person, filming the participant's back from behind (FIG. 2a). Participants thus saw their body from an 'outside', third-person perspective. In one study using this approach ${ }^{60}$, subjects viewed the video image of their body (the 'virtual body') while an experimenter stroked their back with a stick. The stroking was thus felt by the participants on their back and also seen on the back of the virtual body. The HMD displayed the stroking of the virtual body either in real-time or not (using an online video-delay or offline pre-recorded data), generating synchronous and asynchronous visuotactile stimulation.

In another study ${ }^{58}$, seated subjects wearing two HMDs viewed a video of their own body, which was being filmed by two cameras placed $2 \mathrm{~m}$ behind their body. Here, the experimenter stroked the subject on the chest with a stick and moved a similar stick just below the camera. The stroking was thus felt by the subject and seen when not occluded by the virtual body (FIG. 2b). 
a

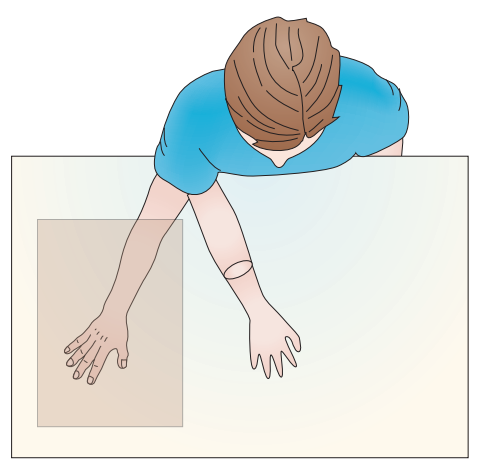

b

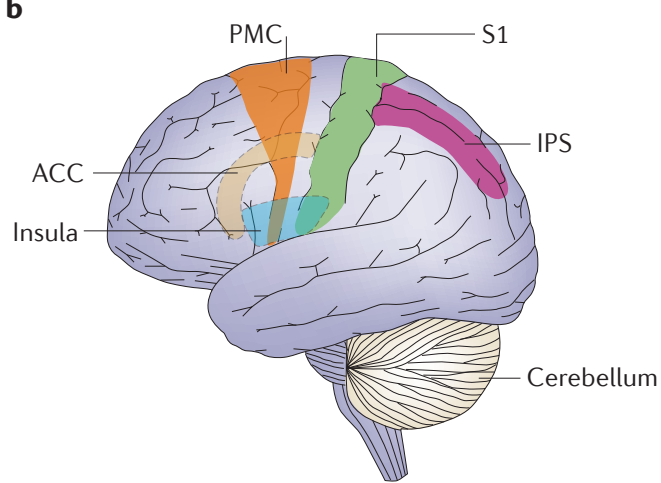

c

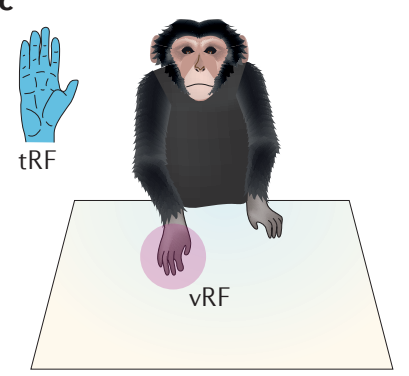

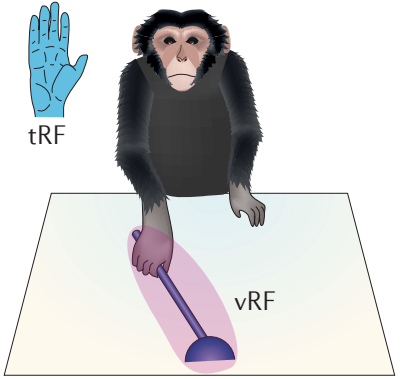

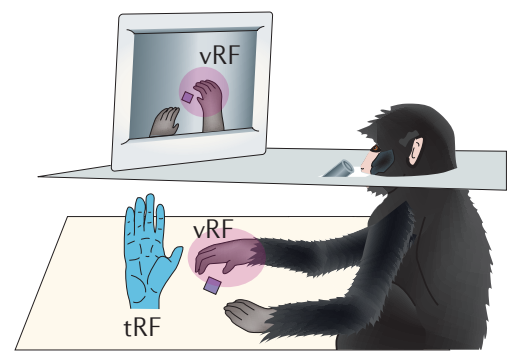

d
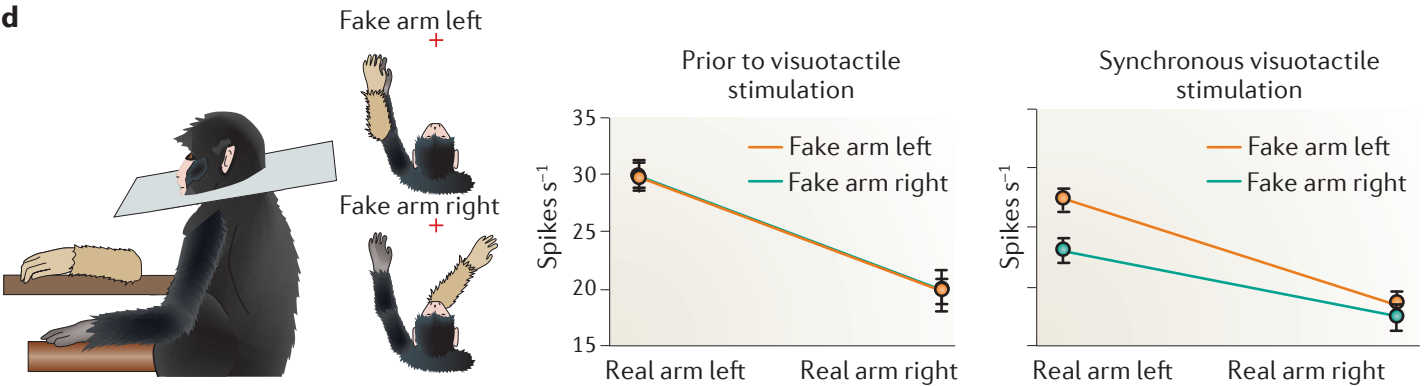

Figure 1 | Illusory hand ownership. a| Experimental set-up of the rubber hand illusion. Participants see a rubber or fake hand (centre) at a plausible distance and orientation from their hand (left), which is hidden from view. Synchronous stroking of both hands (at corresponding locations and with the same speed) leads to the illusion that the touch is felt on the seen rubber hand, accompanied by a feeling of ownership for the rubber hand and a change in perceived hand position towards the rubber hand (a phenomenon known as proprioceptive drift). Asynchronous stroking, implausible location and incongruent orientation of the rubber hand with respect to the participant's hand abolish the illusion. b| The main brain regions that are associated with illusory hand ownership and changes in perceived hand position. Regions include the ventral and dorsal premotor cortex (PMC), primary somatosensory cortex (S1), intraparietal sulcus (IPS), insula, anterior cingulate cortex (ACC) and the cerebellum. $\mathbf{c}$ Receptive fields of bimodal neurons in the IPS region of macaque monkeys that respond to tactile and visual stimulation. The left panel shows the tactile receptive field (tRF; blue) and the visual receptive field (vRF; pink) of a bimodal (visuotactile) neuron that responds to touches applied to the indicated skin region and to visual stimuli presented in the indicated region of visual space surrounding the arm and hand. The size of the vRFs can be extended to more distant locations through tool use (middle panel). Similar extensions of vRFs have been observed when vision of the hand and arm is not mediated by direct sight but mediated via video recordings (right panel). d | Trimodal neurons in the IPS region of macaque monkeys respond to visual, proprioceptive and tactile stimulation. For such neurons, the position of the visual receptive field remains fixed to the position of the arm across several different postures and is based on proprioceptive signals about limb position. The left panel shows an experimental set-up (with the hidden arm positioned below the fake arm) that has been used to reveal that such neurons also respond to tactile and visuotactile stimulation. The activity of such neurons can be altered by visuotactile stroking applied to the fake hand and the hidden hand of the animal. Before visuotactile stroking, the neuron showed greater firing when the real arm was positioned to the left than when it was positioned to the right, but the position of the fake arm did not affect its firing rate (middle panel). After synchronous stroking, but not asynchronous stroking (not shown), the neuron was sensitive to the position of both the real arm and the fake arm (right panel). This suggests that such trimodal neurons can learn to encode the fake arm's position. Part c is modified, with permission, from REF. 43 C (2004) Elsevier and REF. $214 @(2001)$ Elsevier. Part d is modified, with permission, from REF. 48 @ (2000) American Association for the Advancement of Science. 
A third study ${ }^{61}$ involved subjects in a supine body position. Their bodies were filmed by a camera placed $2 \mathrm{~m}$ above the subject so that the virtual body, seen on an HMD, appeared to be located below the physical body. Here, the subjects received both back and chest stroking (although not simultaneously) and saw the virtual body receiving the same type of stroking.

Studies using these types of set-ups to target selfidentification, self-location and the first-person perspective are the focus of the following sections.

\section{Self-identification}

Experimentally induced changes in self-identification. In the study in which subjects viewed the video image of their body while an experimenter stroked their back with a stick ${ }^{60}$ (FIG. 2a), illusory self-identification with the virtual body and referral of touch were stronger during synchronous than during asynchronous stroking ${ }^{60}$, similar to the rubber hand illusion ${ }^{20}$. In the second study ${ }^{58}$, in which seated subjects were stroked on the chest (FIG. 2b) while they viewed their body from behind, the subjects also reported referral of touch (the feeling that the stick they saw was touching their real chest). They also reported that during synchronous stroking, looking at the virtual body was like viewing the body of someone else (that is, they had low self-identification with the virtual body). In the third study ${ }^{61}$, subjects in a supine position saw their virtual body (on an HMD), which appeared to be located below the physical body. Here, self-identification with and referral of touch to the virtual body were greater during synchronous than during asynchronous back stroking. By contrast, self-identification with the virtual body was lower during synchronous chest stroking as compared to asynchronous chest stroking.

Unlike older studies ${ }^{62-66}$ (FIG. 2C), these recent studies have the advantage that self-identification can be tested experimentally across well-controlled conditions of visuotactile stimulation while keeping motor and vestibular factors constant. It has also been shown that illusory full-body self-identification is associated with an interference of visual stimuli on the perception of tactile stimuli ${ }^{67,68}$ (FIG. 2d). Such visuotactile interference is a behavioural index of whether visual and tactile stimuli are functionally perceived to be in the same spatial location $^{67,69-72}$. These findings suggest that during illusory self-identification, visual stimuli seen at a position that is $2 \mathrm{~m}$ in front of the subject's back, and tactile stimuli that were applied on the subject's back were functionally perceived to be in the same spatial location (also see REFS 67,69-74).

Illusory self-identification with a virtual body is also associated with physiological and nociceptive changes. Thus, the skin conductance response to a threat directed towards the virtual body ${ }^{44,58,75}$ as well as pain thresholds (for stimuli applied to the body of the participant during the full-body illusion) ${ }^{76}$ are increased in states of illusory self-identification. The changes in touch, pain perception and physiology that occur during illusory self-identification indicate that states of illusory self-identification alter the way humans process stimuli from their body.
Activity in cortical areas reflects self-identification. Three imaging studies on self-identification have been carried out to date. They all manipulated self-identification through visuotactile stimulation, although they differed greatly in terms of the experimental set-up. One comprehensive fMRI study ${ }^{44}$ of a full-body illusion reported that self-identification with a virtual body is associated with activity in the bilateral ventral PMC, left IPS and left putamen (FIG. 3a). The activity in these three regions was enhanced by visuotactile stimulation when the virtual body was seen in the same place as the participant's body (from a first-person viewpoint and not in back-view; see below). Activity in these regions was also enhanced when visuotactile stimulation was applied to the virtual hand and the subject's corresponding (hidden) hand ${ }^{44}$.

An electroencephalography (EEG) study ${ }^{77}$ linked self-identification with a virtual body to activity in bilateral medial sensorimotor cortices and medial PMC (FIG. 3a). Specifically, self-identification (and selflocation) with a virtual body induced by synchronous versus asynchronous visuotactile stimulation of the real and the virtual body was associated with differential suppression of alpha band power $(8-13 \mathrm{~Hz})$ oscillations in bilateral medial sensorimotor regions and the medial $\mathrm{PMC}^{77}$. These changes in alpha band suppression between synchronous versus asynchronous stimulation conditions were absent if a virtual control object was used instead of a virtual body. Alpha band oscillations over central areas (that is, the mu rhythm) have been linked to sensorimotor processing ${ }^{78}$, and $\mathrm{mu}$ rhythm suppression is thought to reflect increased cortical activation in sensorimotor and/or premotor cortices $^{79}$. Indeed, movements, movement observation ${ }^{80}$, motor imagery ${ }^{81}$ and biological motion perception ${ }^{82}$ suppress mu oscillations in the sensorimotor cortex, as do the application of tactile cues ${ }^{83}$ and the observation of touch applied to another person ${ }^{84}$. These EEG data thus suggest increased activation of the sensorimotor cortex and PMC during asynchronous, as compared to synchronous, visuotactile stimulation. This is similar to findings from a PET study of illusory hand ownership ${ }^{33}$ but opposite to the increased BOLD (bloodoxygen-level-dependent) activity found during the synchronous stroking condition in the fMRI study ${ }^{44}$.

A second fMRI study ${ }^{59}$ found that self-identification with a virtual body is associated with activation in the right middle-inferior temporal cortex (partially overlapping with the extrastriate body area (EBA)) (FIC. 3a). The EBA is, like the PMC and IPS, involved in the processing of human bodies ${ }^{85-88}$. More work is needed as only three neuroimaging studies have been carried out to date, and the results and the applied methods vary greatly.

Self-identification and multisensory integration. The bilateral PMC, IPS and sensorimotor cortex have also been associated with illusory limb ownership, suggesting that full-body and body-part ownership may, at least partly, recruit similar visuotactile mechanisms and similar brain regions ${ }^{44}$. Findings from non-human primates suggest that self-identification for an arm and 


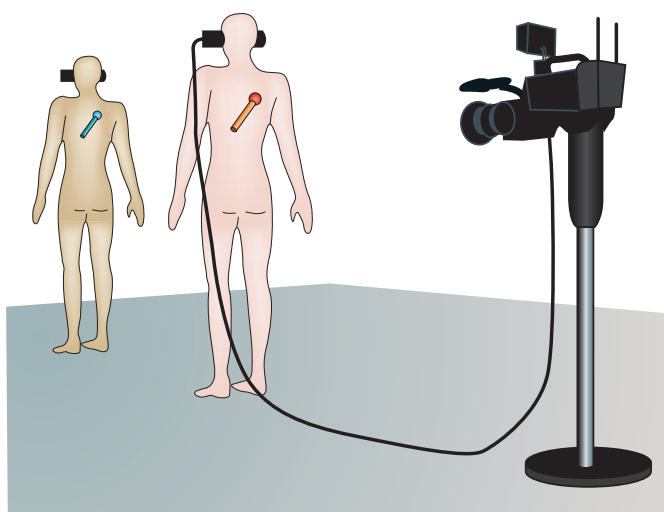

C

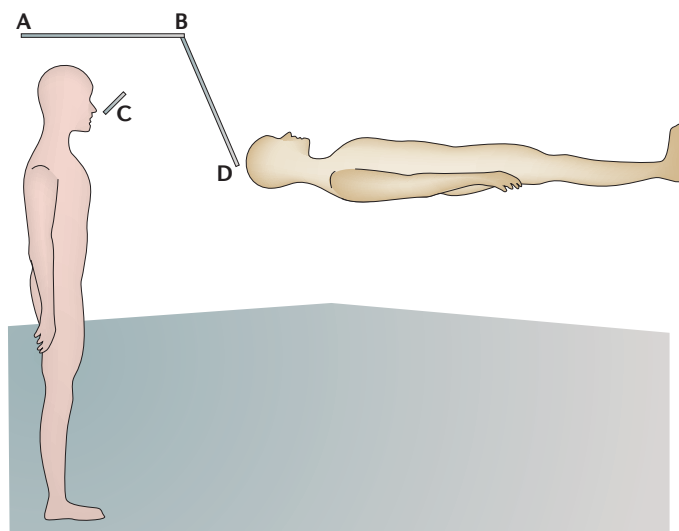

b

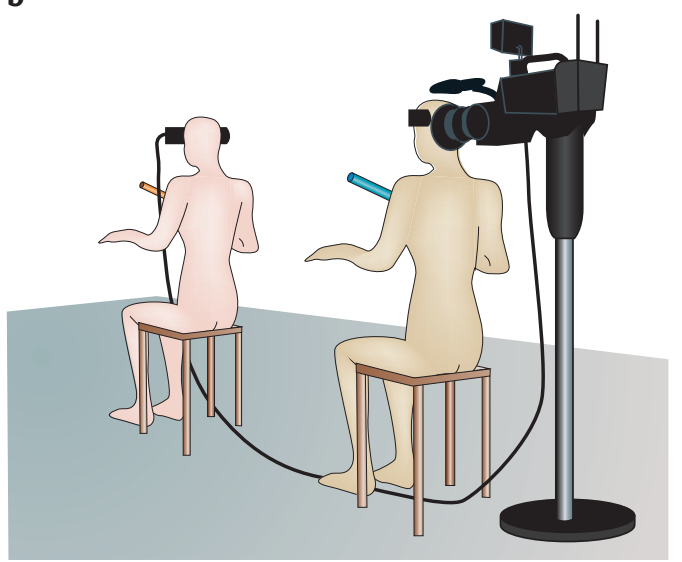

d

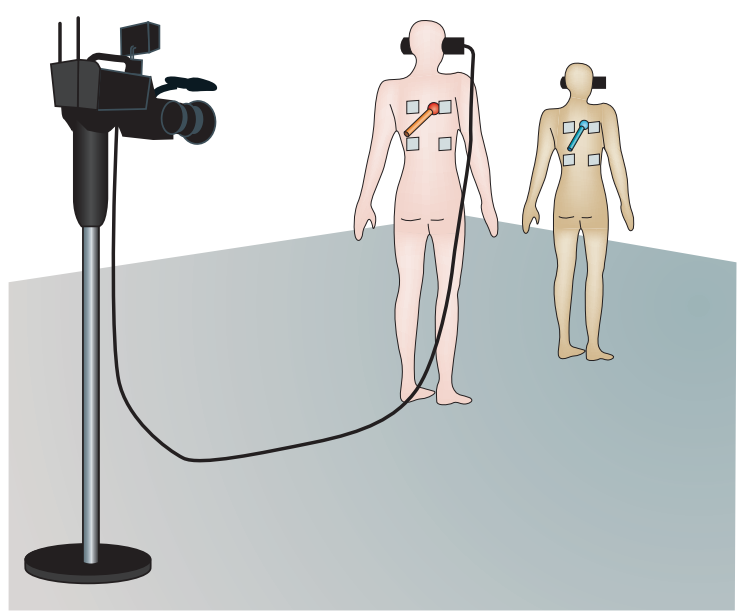

Figure 2 | Set-ups of illusory self-identification experiments. a | Experimental set-up during the full-body illusion using back stroking ${ }^{60}$. A participant (light colour) views, on video goggles, a camera recording of his own back, as if a virtual body (dark colour) were located a few metres in front. An experimenter administers tactile stroking to the participant's back (stroking stick; red colour), which the participant sees on the video goggles as visual stroking on the virtual body. Synchrony (real-time projection) but not asynchrony (pre-recorded or delayed projection) of visuotactile stroking results in illusory self-identification with the virtual body. $\mathbf{b}$ | Experimental set-up during the full-body illusion using chest-stroking ${ }^{58}$. An experimenter applies simultaneously tactile strokes (unseen by the participant) to the chest of the participant (light colour) and visual strokes in front of the camera, which films the seated participant from a posterior position. On the video goggles, the participant sees a recording of the own body, including the visual strokes, from the posterior camera position. Synchronous (real-time video projection) but not asynchronous (delayed video projection) visuotactile stroking results in illusory self-identification with the camera viewpoint (represented by the body in the dark colour). $\mathbf{c}$ An early experimental set-up using a portable mirror device is shown, in which several aspects of bodily self-consciousness, likely including self-identification, were manipulated. Four portable mirrors (A-D) were aligned around a participant (standing position) in such a way that the participant could see in front of him a visual projection of his body in a horizontal position. $\mathbf{d}$ | The experimental set-up of a full-body illusion using back stroking (a) has also been used to acquire repeated behavioural measurements related to visuotactile perception (that is, the crossmodal congruency effect (CCE) $)^{68}$. In addition to the visuotactile stroking (as in a) participants wore vibrotactile devices and saw visual stimuli (light-emitting diodes) on their back while viewing their body through video goggles. The CCE is a behavioural measure that indicates whether a visual and a touch stimulus are perceived to be at identical spatial locations. Participants were asked to indicate where they perceived a single-touch stimulus (that is, a short vibration), which was applied either just below the shoulder or on the lower back. Distracting visual stimuli (that is, short light flashes) were also presented on the back either at the same or at a different position (and were filmed by the camera). Under these conditions, participants were faster to detect a touch stimulus if the visual distractor was presented at the same location (that is, a congruent trial) compared to touches co-presented with a more distanced visual distractor (that is, an incongruent trial). CCE measurements were carried out while illusory selfidentification was modulated by visuotactile stroking as described in part $\mathbf{a}$. The effect of congruency on reaction times was larger during synchronous visuotactile stroking than during asynchronous stroking, indicating greater interference of irrelevant visual stimuli during illusory self-identification with the virtual body. Part c is modified, with permission, from REF. 65 (c) (1899) Oxford University Press. 
for a full-body both rely on visuotactile neurons. For example, the PMC and IPS in non-human primates harbour bimodal neurons that are involved in integration of visual and somatosensory stimuli regarding the arms and the trunk $\mathrm{k}^{38,41,43,48}$. Thus, in addition to arm-centred neurons (see above), these regions harbour trunk-centred neurons that have large receptive fields ${ }^{43}$ (FIG. 3b): that is, they encode the surface of the trunk $\mathrm{k}^{38,89,90}$ and, in some cases, the whole body of the monkey ${ }^{89}$. On the basis of the involvement of the IPS and PMC in humans in both hand ownership and self-identification (that is, body ownership) and the properties of bimodal visuotactile neurons in these regions in monkeys, it can be speculated that changes in full-body self-identification may be a result of strokinginduced changes in the size and position of trunk- centred bimodal neurons with respect to the virtual body that is seen on the HMD. In this scenario, the visual receptive fields of such bimodal neurons would be enlarged following visuotactile stroking, and would also encode the more distant position of the seen virtual body after stroking ${ }^{43}$ (FIG. 3C).

However, there are also some important differences between full-body and body-part ownership. For example, during the full-body illusion, there is self-identification with a virtual body that is viewed at a distance of $2 \mathrm{~m}$, whereas in the rubber hand illusion, the illusion decreases or disappears when the rubber hand is placed at more distant position $\mathrm{s}^{25}$ or when the posture of the rubber hand is changed to an implausible one $e^{23}$. Considering that viewing one's body from an external perspective at $2 \mathrm{~m}$ distance is even less anatomically a

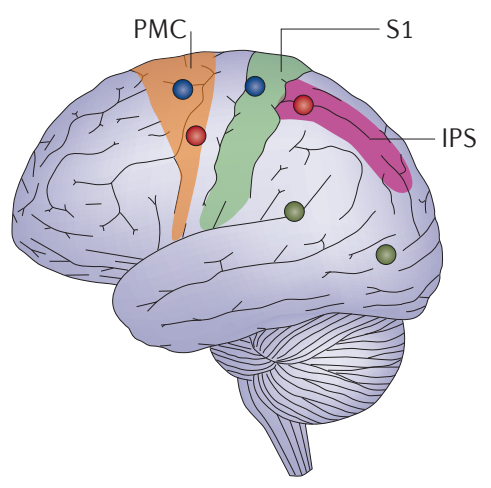

b

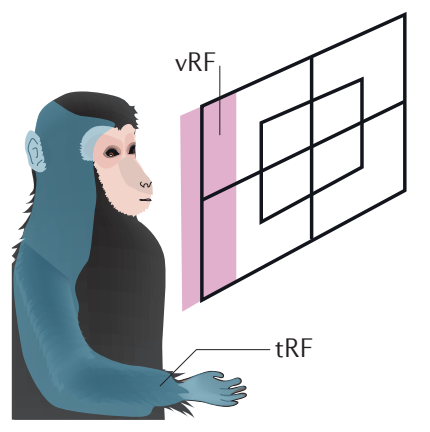

c Prior to visuotactile stimulation

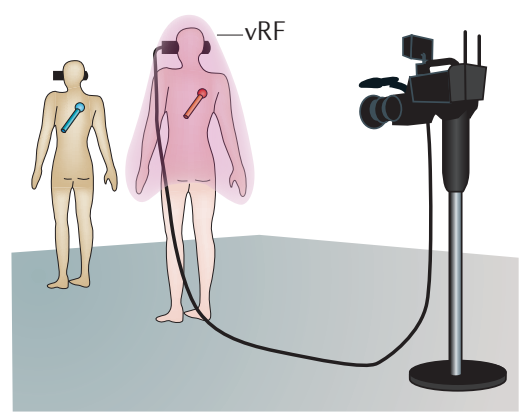

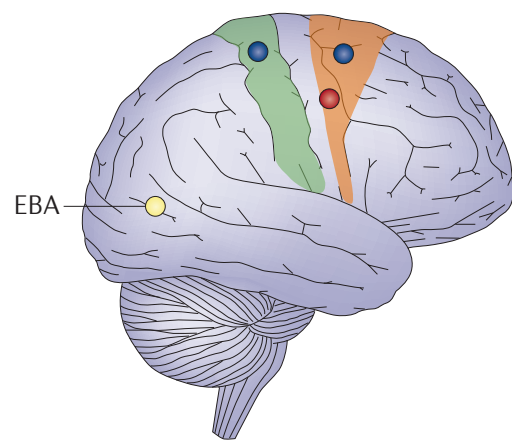

Figure 3 | Brain mechanisms of illusory self-identification. a|The drawing shows the different brain regions that have been implicated in illusory self-identification. Regions include the ventral premotor cortex (VPMC), primary somatosensory cortex (S1), intraparietal sulcus (IPS), extrastriate body area (EBA) and the putamen (not shown). Data by Petkova et al..$^{44}$ are shown in red, by Lenggenhager et al..$^{77}$ in blue and by lonta et al. ${ }^{59}$ in yellow. The location of brain damage leading to heautoscopy is also shown ${ }^{98}$ (green). b | Receptive fields of bimodal neurons in area VIP (ventral intraparietal) of macaque monkeys that respond to both tactile and visual stimulation. In both panels, the size and position of the tactile receptive field (tRF) is indicated in blue and the size and position of the visual receptive field (vRF) in peripersonal space is indicated in pink. A neuron in area VIP responds to tactile stimuli applied to a large skin region encompassing the right shoulder, right arm and right half of the head, and to visual stimuli from the large visual region indicated in pink (left panel). Other neurons in area VIP respond to tactile stimuli applied to the entire trunk and the right arm (tRF; blue) $)^{90}$ and visual stimuli in the upper bilateral visual fields (vRF; pink) (right panel). Other neurons (not shown) respond to tactile stimuli applied to the right hemibody and visual stimuli from the entire right visual field (vRF). Note the congruence of the size and location of vRFs and tRFs for each neuron and the larger size of the RFs with respect to arm- or hand-centred bimodal neurons depicted in FIG. 1 c. Neurons with similar properties have also been described in area 5 and the PMC.c|Hypothetical changes in the size and/or position of the vRF of trunk-centred bimodal VIP neurons that may be associated with illusory self-identification during the full-body illusion as induced by visuotactile stroking between the participant's body (light-coloured body) and the filmed (dark-coloured) body (also see FIG. 2a). The left panel shows the bilateral vRF (in pink) of a bimodal visuotactile neuron that responds to stimuli that are seen as approaching the person's arms, trunk and the back of the head (location of tRFs not shown). During the full-body illusion, the sight of one's own body filmed from behind and viewed through a head-mounted display may alter the size and/or position of the vRFs of such trunk-centred visuotactile neurons, so that they now extend to the more distant position of the seen filmed body (right panel). Such vRF changes in the full-body illusion may be particularly prominent under conditions of synchronous visuotactile stroking applied to the filmed back and the hidden back of the subject, as shown for visuotactile stroking between a participant's hidden hand and a fake hand ${ }^{48}$. 
Heautoscopy

The phenomenon in which the subject experiences seeing a second own-body in extracorporeal space. Subjects often report strong self-identification with the second own-body and heautoscopy is often associated with the sensation of bi-location (that is, the sensation of being at two places at the same time). plausible than a fake hand in a misaligned posture, it is perhaps surprising that the full-body illusion occurs at all under such conditions (but see REF. 91). I argue that further differences between trunk- versus arm-centred neurons may account for this. Thus, in monkeys, the visual receptive fields of bimodal neurons with tactile receptive fields that are centred on the trunk (including the back and shoulder) in area 5 (REF. 43) and area VIP (ventral intraparietal) $)^{90}$ in the parietal cortex are larger than those of neurons with hand-centred visual and tactile receptive fields (FIG. 3b). Moreover, the visual receptive fields of trunk-centred visuotactile neurons sometimes extend for $1 \mathrm{~m}$ or more into extrapersonal space $^{43}$, whereas the visual receptive fields of armcentred visuotactile neurons extend less far ${ }^{42,43}$ (for trunk-centred bilateral neurons in area 5, see REFS 92,93).

Thus, although arm and full-body ownership are both associated with visuotactile mechanisms in the sense that the neurons involved respond to both visual and tactile stimuli and depend on the temporal congruency between seen and felt stimulation, they probably rely on at least partly different mechanisms, as trunkand hand-centred visuotactile neurons differ in the location and the size of their visual and tactile receptive fields $s^{38,40-43,48,92}$. In addition, trunk- versus hand-centred visuotactile neurons are likely to be found within different subregions involved in visuotactile integration (their location differs, for example, in area 5, although this has so far only been described for tactile neurons ${ }^{92,93}$ ). Moreover, area VIP has more visuotactile trunk- and head-coding cells than hand-coding cells, whereas the opposite is true for more anterior areas in the IPS ${ }^{94}$ and area 5. Although visuotactile neurons have not been defined in the $\mathrm{EBA}^{59}$, it can be speculated that the cellular mechanisms for self-identification in the EBA are similar because activity in this region is modulated by movements of the observer ${ }^{85}$ as well as during tactile explorations of body-shaped objects ${ }^{95,96}$.

Neurologically induced changes in self-identification. Patients with heautoscopy ${ }^{1,97}$ report strong changes in self-identification with a hallucinated visual body. These patients report seeing a second own-body in extrapersonal space and often self-identify and experience a close affinity with this autoscopic body ${ }^{56,97,98}$. Self-identification with the hallucinated body may even persist if the hallucinated body only partly reflects the patient's outside bodily appearance ${ }^{97,98}$, which is compatible with illusory self-identification that can be induced with avatars and fake bodies that do not resemble the body of the participant ${ }^{44,59,60,75}$. Heautoscopy is associated with vestibular sensations and detachment from emotional and bodily processing from the physical body, suggesting links with depersonalization disorder ${ }^{97,99}$. It has been proposed that heautoscopy is a disorder of multisensory (in this case, visual, tactile and proprioceptive) integration of bodily signals and an additional disintegration of such cues with vestibular signals ${ }^{100}$. Patients with heautoscopy do not just report abnormalities in self-identification but also in self-location (see below). To the question "where am I in space?" they cannot provide a clear answer, and self-location may frequently alternate between different embodied and extrapersonal positions and may even be experienced at two positions simultaneously ${ }^{14,97,100,101}$. This experience may sometimes be described as if being "split in two parts or selves", as if "I were two persons" (REF. 102) or as having a "split personality" (REF. 103). Although the precise location of brain lesions that induce heautoscopy has not yet been identified, a recent review suggests a predominant involvement of the left temporoparietal cortex and to a lesser extent the occipitotemporal cortex ${ }^{98}$ (FIG. 3a).

Collectively, the data reviewed above suggest that self-identification is linked to activity in five cortical regions - the IPS, PMC, sensorimotor cortex, EBA and temporoparietal cortex - and probably also in subcortical structures like the putamen. The EBA, sensorimotor cortex and temporoparietal cortex were less consistently observed across the reviewed data, suggesting that IPS and PMC processing is most important. These five cortical areas are known to integrate multisensory bodily signals - including visual, somatosensory and vestibular signals ${ }^{38,41-43,90,104}$ - and all except the EBA and sensorimotor cortex have been shown to harbour bimodal (or multimodal) neurons (for multimodal neurons in the temporoparietal junction (TPJ), see next section) that have large receptive fields encompassing the trunk and face region and, in some cases, the legs. Experimentally induced changes in illusory self-identification with a fake or virtual body via video-based virtual reality systems may be associated with a stroking-induced enlargement or alteration of the visual receptive fields of such bimodal neurons (FIG. 3c) in these five areas (especially the IPS and PMC), although no direct evidence for this possibility exists yet. More neuroimaging work in humans is necessary to better understand the different activation patterns across studies and how they relate to differences in visuotactile stimulation paradigms and self-identification.

\section{Self-location and first-person perspective}

Under normal conditions, in the described laboratory conditions, and in most reports by neurological patients, the position of self-location and the first-person perspective coincide, and changes in self-location and firstperson perspective are therefore described together here. In rare instances, however, self-location and first-person perspective can be experienced at different positions ${ }^{105}$, suggesting that it may be possible to experimentally induce similar dissociations in healthy subjects.

Experimentally induced changes in self-location and first-person perspective. Attempts to study self-location in healthy individuals through self-reports ${ }^{106,107}$, interviews, pointing ${ }^{108}$ and schematic drawings ${ }^{109}$ found that most participants indicated self-location within their body, particularly in the head. Can alterations in selflocation be induced experimentally? Stratton reported heautoscopy-like changes in self-location as early as 1899 (REF. 65) (FIG. 2c). In an observational study ${ }^{63}$, the authors installed a fixed camera in the corner of a room and projected the filmed scene (including the subject's 
Ego-centre human observers believe they are viewing a spatial scene. Ego-centres have been investigated for visual, auditory or kinaesthetic stimuli.
A single point from which

body) onto their subject's HMD so that they could see their body from a distance while walking. Using such sensorimotor cues, subjects reported being both at the position of the camera and at the position at which they saw their body. More recently, researchers have induced alterations in self-location by employing the techniques

a
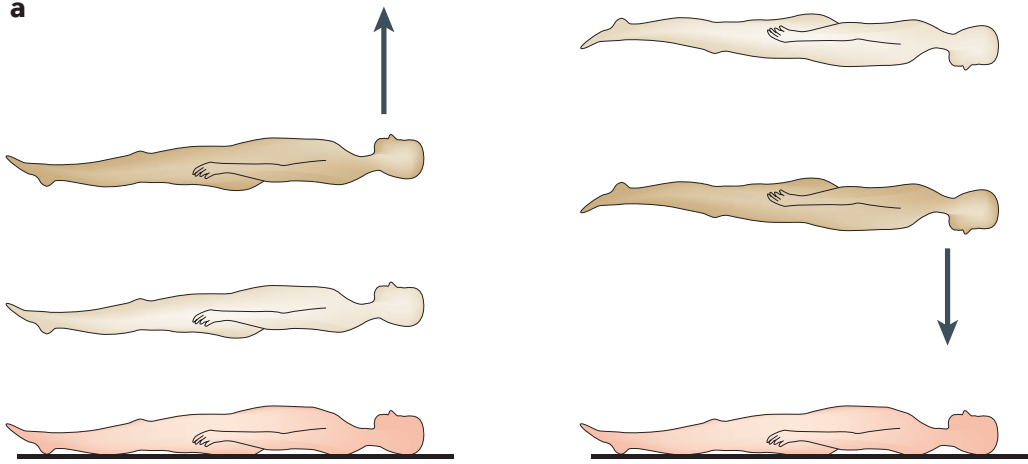

b

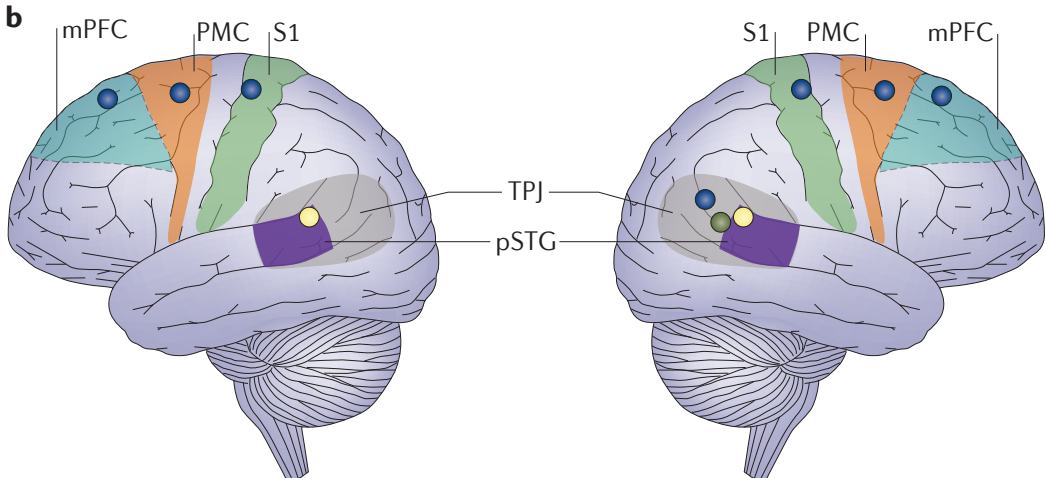

Figure 4 | Illusory self-location and first-person perspective. a |Self-location and first-person perspective depend on visuotactile signals and their integration with vestibular signals. The left panel shows a participant lying horizontally on her back (pink body) and receiving back stroking from a robotic stimulator (not shown) installed on the bed. While she receives such tactile stimulation, she is watching (on video goggles) a video of another person receiving the back stroking (body not shown). Under this visuotactile condition, one group of participants experienced looking upward (upward first-person perspective) associated with elevated self-location, and this experience was stronger during synchronous stroking (left panel, dark body) than during asynchronous stroking condition (left panel, beige body). Another group of participants, who received physically identical visuotactile stimulation conditions, experienced looking downward associated with lower self-location, and this experience was also stronger during synchronous stroking (right panel, dark body) than during asynchronous stroking (right panel, beige body). These differences in self-location and experienced direction of the first-person perspective are probably due to different weighing of visual and vestibular cues related to gravity perception. Thus, the visual cues from the posture of the filmed body suggested that the direction of gravity is upward, while the veridical direction of gravity is always downwards. Participants in the left panel seem to rely more strongly on vestibular versus visual cues, whereas the opposite is true for participants depicted in the right panel, when judging self-location and the direction of the first-person perspective. The direction of the experienced direction of the first-person perspective is indicated by an arrow in both panels. $\mathbf{b}$ |The drawing shows the different brain regions that were activated during illusory self-location and changes in the first-person perspective in different studies. Regions include the right and left posterior superior temporal gyrus (pSTG), right temporoparietal junction (TPJ), primary somatosensory cortex (S1) and medial premotor cortex (mPMC) and adjacent medial prefrontal cortex (mPFC). Data by Lenggenhager et al. ${ }^{77}$ are shown in blue, data by lonta et al..$^{59}$ are shown in yellow and the location of brain damage at the right angular gyrus that leads to out-of-body experiences is shown in green ${ }^{59}$. that are used to study self-identification, which are described above (FIC. 2). Results from these studies ${ }^{58,60}$ indicated that during the illusion, subjects experienced self-location (measured by questionnaires ${ }^{58}$, walking responses $^{60}$ or mental imager $y^{59,61}$ ) not at the position of their physical body but either 'in front of' or 'behind' that position, depending on whether the actual and virtual body received synchronous back stroking ${ }^{60}$ or chest stroking ${ }^{58}$. Comparable changes in self-location occurred when subjects were in supine position ${ }^{61}$ (FIG. 4a, left panel).

In a recent $\mathrm{fMRI}$ study ${ }^{59}$, participants in a supine position viewed, through video goggles, short movies showing a back view of a virtual body that was filmed from an elevated position (that is, by a camera positioned above the virtual body). The participant received back strokes (robotic stroking) while viewing the video, and these were either synchronous or asynchronous with the back strokes that the virtual body received on the video. Subjects reported higher self-location towards the virtual body during the synchronous compared with the asynchronous stroking condition (FIG. 4a). Participants were also asked to indicate the experienced direction of their first-person perspective (either upwards or downwards). Interestingly, despite identical visuotactile stimulation, half of the participants experienced looking upward towards the virtual body (up-group) and half experienced looking down on the virtual body (down-group). Importantly, these changes in first-person perspective were associated with different changes in self-location in both groups: up-group participants reported an initially low position of self-location and an elevation in self-location during synchronous stroking, whereas participants from the down-group reported the opposite (FIG. 4a). Moreover, subjective reports of elevated self-location and sensations of flying, floating, rising, lightness and being far from the physical body were frequent in the down-group and rare in the up-group ${ }^{59}$. These data show, first, that self-location depends on visuotactile stimulation and on the experienced direction of the first-person perspective. Second, these data suggest that different multisensory mechanisms underlie self-location versus self-identification, as the latter does not depend on the first-person perspective ${ }^{59}$. Different multisensory mechanisms have also been described for illusory hand ownership and perceived hand location in the rubber hand illusion paradigm ${ }^{28,30}$, which can be compared with illusory self-identification and self-location, respectively.

It is currently not known whether and how these experiences of self-location and the first-person perspective relate to those in earlier studies on the visual, auditory and kinesthetic ego-centre and to subjective reports based on interviews and pointing ${ }^{108,109}$. It should be of interest to test whether the visual ego-centre ${ }^{110}$ can be altered through visuotactile stimulation and, if so, whether such changes are body-specific and depend on visuotactile synchrony. Self-location and the first-person perspective as manipulated through visuotactile stimulation may also relate to brain mechanisms of prism adaptation. Prism adaptation is generally studied by inserting 


\section{Prism adaptation \\ The phenomenon that subjects who wear prism glasses that introduce spatial mismatches between the seen position of visual cues and their actual spatial coordinates learn to correctly perceive and reach for visual targets.}

\section{Out-of-body experience}

(OBE). The phenomenon in which the subject experiences seeing a second own-body from an elevated and distanced extracorporeal position. Subjects often report disembodiment (that is, a sensation of separation from their physical body) and sensations of flying and lightness.

\section{Virtual mirrors}

Part of an immersive virtual reality scenario that includes a region where the image and movements of the immersed user will be simulated as if reflected from a physical mirror.

\section{Egocentric}

An umbrella term for maps and/or patterns of modulation that can be defined in relation to some point on the observer (for example, head- or eye-centred maps). systematic spatial mismatches between the seen position of visual cues and their actual spatial coordinates ${ }^{11-115}$. The recent experiments on self-location and the firstperson perspective described above may thus be conceived as a form of prism adaptation that uses a complex displacement of the visuospatial field and the position of the observer within it. Future research should investigate whether experimentally induced changes in self-location and first-person perspective rely on similar mechanisms to those described for prism adaptation ${ }^{111-115}$.

Activity in bilateral temporoparietal cortex reflects selflocation and first-person perspective. In an EEG study ${ }^{77}$, modulation of self-location was associated with $8-13 \mathrm{~Hz}$ oscillatory activity in bilateral medial sensorimotor and medial PMC (FIG. 4b). In addition, gamma band power in the right TPJ and alpha band power in the medial prefrontal cortex correlated with the strength of the induced changes in illusory self-location. An fMRI study also showed an association between changes in selflocation and first-person perspective and activity at the TPJ bilaterally ${ }^{59}$. Here, TPJ activity, which peaked in the left and right posterior superior temporal gyrus (pSTG), differed between synchronous and asynchronous stroking conditions (FIG. 4b), and, importantly, depended on the experienced direction of the first-person perspective ${ }^{59}$. Thus, in one group of subjects, pSTG activity was higher in the asynchronous stroking condition, whereas in another group of subjects PSTG activity was higher in the synchronous stroking condition - that is, the BOLD response was smaller during conditions in which subjects from either group experienced an elevated self-location ${ }^{59}$. The finding in this fMRI study that selflocation depended on the first-person perspective shows that the matching of different sensory inputs alone does not account for pSTG activity in healthy subjects.

Neurologically induced changes in self-location and first-person perspective. The involvement of the PSTG in self-location and the first-person perspective is consistent with out-of-body experiences (OBEs) in patients with damage to the pSTG. These patients experience a change in both self-location and first-person perspective - they see and/or feel their body and the world from an elevated perspective that does not coincide with the physical position of their body $98,100,116$. Although this first-person perspective is illusory, it is experienced in the same way as humans experience their everyday first-person perspective under normal conditions ${ }^{117-119}$. This phenomenon has been induced experimentally in a patient with epilepsy who experienced OBEs ${ }^{120}$ that were characterized by elevated self-location and a downwardlooking first-person perspective by applying $2 \mathrm{~s}$ periods of electrical stimulation at the anterior part of the right angular gyrus and the pSTG. For $2 \mathrm{~s}$ periods, this patient experienced the sensation of being under the ceiling and seeing the entire visual scene (including the room, her body and other people) from her stimulation-induced elevated first-person perspective and self-location. The findings from the experiment using robotic stroking ${ }^{59}$ described above are intriguing in this respect, as they showed that under certain experimental conditions, healthy subjects can experience a $180^{\circ}$ inversion and displacement of the first-person perspective similar to the perspective changes seen in patients with OBEs. On the basis of other hallucinations that are associated with OBEs - including vestibular otolithic sensations (such as floating, flying and elevation) and visuotactile hallucinations ${ }^{100,105,120-122}$ - it has been proposed ${ }^{98}$ that OBEs are caused by abnormal integration of tactile, proprioceptive, visual and in particular vestibular inputs. Anatomically, OBEs resulting from focal brain damage or electrical brain stimulation have been associated with many different brain structures ${ }^{100,120,123,124}$ but most often involve the right angular gyrus ${ }^{59}$ (FIC. 4b).

Viewpoint changes and spatial navigation. The search for the brain mechanisms underlying the first-person perspective and its relation to other aspects of self-consciousness has been approached from many different angles (see below) ${ }^{98,125}$. However, these studies focused on imagined or visual changes in the first-person perspective versus third-person viewpoints that differ from the changes in the experienced direction of the firstperson perspective described above in neurological and healthy subjects. For example, some experiments have studied self-identification by changing the viewpoint from which a virtual body was shown. Thus, one study tested whether participants experienced differences in self-identification depending on whether they saw a virtual body from a first- versus third-person viewpoint ${ }^{126}$ (also see REF. 75). In the first-person viewpoint condition, participants tilted their heads down as if to look towards their stomach while being shown the stomach and legs of a virtual body on an HMD. In the thirdperson viewpoint condition, participants were asked to look straight ahead and saw a front-facing virtual body at a short distance. The participants reported higher self-identification for first- versus third-person viewpoints $^{126}$ (also see REF. 127). Higher self-identification with a virtual body was also reported by supine subjects who received stroking and simultaneously watched synchronous (as compared to asynchronous) stroking being applied to a virtual body that was seen as if in the same place as their own physical body (first-person viewpoint $)^{44}$. Activity the in left and right PMC and in the left IPS was increased in conditions with higher levels of self-identification ${ }^{44}$. Findings from a study in which participants observed and interacted with virtual humans, virtual mirrors and other virtual objects ${ }^{127}$ confirmed the importance of the first-person viewpoint for the strength of self-identification with a virtual body, but also showed that under the first-person viewpoint visuotactile stimulation did not strongly alter self-identification, whereas it did for third-person viewpoints. Together, these data show that different visual viewpoints of a virtual body induce different levels of self-identification and that these may ${ }^{126}$ or may not ${ }^{127}$ depend on visuotactile conflict.

These studies echo recent work that compared different types of 'egocentric' viewpoint transformations and judgements. In several experiments, subjects watched a 
Allocentric

An umbrella term for maps and/or patterns of modulation that are defined in relation to an object external to the observer.

Microgravity environment Environments in which no gravity exists for short periods (parabolic flight) or prolonged periods (orbital flight). virtual scene showing an avatar in the centre and a different number of red balls around her ${ }^{128-130}$. The subjects observed the scene from different viewpoints (including that of the avatar and different red ball positions) and had to either imagine how many balls the avatar could 'see' (that is, an imagined third-person viewpoint) or how many balls they were seeing (that is, a first-person viewpoint). The third-person viewpoint condition was associated with activation in the superior parietal lobule and premotor regions, whereas the first-person viewpoint condition activated the prefrontal cortex, medial posterior parietal cortex and bilateral STG. In a related experiment, frequent viewpoint changes (as compared to a fixed viewpoint) were associated with right TPJ activation (centring on the posterior middle temporal gyrus) ${ }^{131}$ (also see REF. 128). The areas of the brain in which activity changes during different first-person viewpoints may be of relevance for recognizing landmarks and during spatial navigation ${ }^{132}$. For example, a network of brain regions consisting of the right TPJ, IPS, precuneus and parahippocampal gyrus ${ }^{133}$ is activated when subjects imagine looking at a spatial environment from a number of different (that is, imagined third-person) viewpoints. Moreover, these brain regions are different from those involved in allocentric scene transformations ${ }^{134}$ (BOX 1).

Vestibular processing and the first-person perspective. The studies discussed above $e^{44,126}$ involved changes in viewpoint: that is, changes in the visual input to the brain while testing bodily self-consciousness. Importantly, this is different from the situation in patients with OBEs and in experiments involving robotically applied stroking $^{59}$, who experience changes in first-person perspective without any changes in the actual visual input. This suggests that the first-person perspective may have a non-visual, vestibular component, and that the firstperson perspective therefore relies, at least partly, on

\section{Box 1 | Egocentric and allocentric mental tranformations}

There is a long tradition in cognitive neuroscience of studying ego- versus allocentric perspective taking and mental imagery. In egocentric paradigms, participants are classically asked to imagine shifting their position and perspective to a new position and perspective in space and make judgements about variable attributes or spatial relations of stimuli from the imagined position and perspective. Egocentric mental imagery has been associated with activation in the right middle temporal gyrus, supplementary motor area, left middle occipital gyrus ${ }^{206,207}$ and in the left temporoparietal junction (TPJ) ${ }^{208}$. Other studies, in which participants were asked to generate egocentric mental imagery by imagining themselves at the position and perspective of visually presented human figures, have reported bilateral (but predominantly right) TPJ activation, as well as bilateral extrastriate cortex activity in proximity to the extrastriate body area ${ }^{98,125}$, extending earlier findings ${ }^{209}$. This type of egocentric imagery was faster and more accurate when performed from an elevated visual viewpoint and was accompanied by stronger activation in the bilateral TPJ when compared to eye-level or lowered visual viewpoints ${ }^{210}$. Classically, these imagined egocentric viewpoints or bodily transformations are compared to imagined allocentric transformations, in which participants are asked to imagine a transformation of the scene, array or object, rather than a transformation of the observer's viewpoint or position. Comparisons between ego- and allocentric transformations in behavioural and brain imaging studies ${ }^{132,207,211,212}$ have revealed that both are distinct brain processes, with allocentric transformations relying more strongly on structures in the right hemisphere, especially in the right posterior parietal cortex ${ }^{132,207,211,212}$. distinct brain mechanisms from those involved in selfidentification, which rely on visual and somatosensory input. It has been argued ${ }^{59}$ that mechanisms underlying the first-person perspective are of a visuovestibular nature. In the study discussed earlier ${ }^{59}$, participants viewed a visual image on the HMD that contained a conflict between the visual gravitational cues of the virtual body and the vestibular gravitational cues experienced by the participant's physical body: the body that was shown in these experiments was presented in a direction that was incongruent with the direction of veridical gravity. The authors argued that this may have caused differences in the experienced direction of the first-person perspective, with participants from the up-group relying more strongly on vestibular cues from the physical body (indicating downward gravity directed towards the physical body) than on visual gravitational cues from the virtual body (indicating downward gravity directed away from the physical body), whereas participants from the down-group show the opposite pattern. Indeed, there are inter-individual differences in the extent of visuovestibular integration, and some subjects may rely more strongly on visual signals and others on vestibular signals ${ }^{135-138}$.

The possibility that the experienced direction of the first-person perspective depends on visuovestibular integration, especially when the subject is in the supine position, is also compatible with the finding that $73 \%$ of OBEs in the general population ${ }^{139}$ and $>80 \%$ of OBEs in neurological patients occur in the supine position ${ }^{98}$. Moreover, the experienced direction of the first-person perspective is strongly altered in a bodily illusion - the inversion illusion - that is prevalent in people with otolithic deficits and in microgravity conditions ${ }^{140-142}$. This illusion occurs in almost all healthy subjects placed in a microgravity environment ${ }^{142,143}$. It is characterized by a $180^{\circ}$ inversion and a down-looking first-person perspective of the subject within a stable extrapersonal space and results from absent or abnormal gravitational signals and abnormal visuovestibular integration. Finally, body position is known to strongly affect visual and vestibular perception $^{144-147}$ (BOX 2).

To summarize, these data collectively suggest that neurologically and experimentally induced changes in the experienced direction of the first-person perspective may be due to abnormal signal integration of otolithic and visual cues $^{59,100,142,148-150}$.

The role of multimodal neurons in self-location and first-person perspective. It is possible that changes in self-location may, like changes in self-identification, involve shifts in the spatial characteristics of trunk-centred bimodal visuotactile neurons, especially in the TPJ and posterior parietal cortex (such as area VIP), but also in the medial PMC and, potentially, EBA. Thus, it can be speculated that the different visuotactile stroking procedures described above displace or enlarge the visual receptive fields of such bimodal neurons, so that they now also encode the more distant position of the seen body (FIG. 5a). In monkeys, it has been shown that visuotactile stroking can change the properties of neurons in 


\section{Box 2 | Gravity and bodily self-consciousness}

Our bodies have evolved in terrestrial gravity and have consequently adapted to constant linear acceleration. Reports from subjects experiencing microgravity show that the absence of this acceleration can trigger a number of illusory own-body perceptions, and this is compatible with internal brain models of gravity ${ }^{147,213}$. Weightlessness can be obtained during prolonged free fall in aircrafts during parabolic flights or in spacecraft in orbit. In parabolic flights, the free fall lasts for about $30 \mathrm{~s}$, whereas in orbital flight one can keep on falling for several months. The data obtained from orbital and parabolic flights show that subjects have persisting sensations of their body or surrounding space being oriented up- or downwards (vertical orientation), even though the subjects understand that up and down are 'meaningless' concepts in microgravity. In addition, a range of bodily illusions has been reported in microgravity, which are likely to be the result of absent or abnormal gravitational signals, multisensory disintegration and top-down influences. The percept of vertical orientation only disappears when vestibular, somatosensory and visual cues are absent (that is, when people are free-floating with their eyes closed ${ }^{142}$ ). Almost all subjects report disorientation, vestibular symptoms or loss of a spatial anchoring under these conditions ${ }^{141}$. The most common own-body illusion in microgravity is the inversion illusion $^{140-142}$, which was first described by Graybiel and Kellogg ${ }^{143}$. This is defined as a feeling of the body being upside-down relative to extrapersonal space (or the room being upside-down relative to the stable observer). According to Lackner ${ }^{142}$, multiple combinations of inversion illusion and room-tilt illusion may occur. The person may feel like he is upside-down while the room is in its normal orientation (for example, the floor of the aircraft is interpreted as being down); the person may feel upright while the room is upside down; and the person may also feel upside-down in an upside-down room. These illusions can be so compelling that the subjects assume an incorrect position when they are preparing themselves for the end of the parabola and have led to major accidents in orbital flights. Last, touch and pressure cues have a strong influence on the inversion illusion, underlining that under conditions of absent or abnormal otholithic cues, the perception of verticality and own-body orientation in space depends not only on vestibular and visual cues but also on somatosensory input.

\section{Vestibular neurons}

Neurons responding to

activation of receptors in the

vestibular labyrinth

(semicircular canals and otolith

organs).

Otoliths

Organs in the vestibular

labyrinth of the inner ear that are sensitive to linear

acceleration and gravity.

Translational signals Otolithic vestibular signals that cause linear acceleration. area 5 and the PMC, from encoding the location of an animal's physical arm before stroking to an encoding of a fake arm after visuotactile stroking ${ }^{41,48}$. The presence of visuotactile neurons in areaVIP, the PMC and TPJ is well established, but this has not yet been investigated for the EBA. Moreover, the role of the EBA in self versus other discrimination and self-location is not completely understood: EBA activity seems to reflect first- versus third-person perspectives in some studies ${ }^{125}$ but not in others $^{151,152}$.

However, the neuroimaging and neurological data reviewed above suggest that there are also differences between brain mechanisms of self-location versus selfidentification. Although the role of the PMC and EBA in self-identification has been shown by several studies, the involvement of the PMC in self-location is still unclear $^{40,59}$ and EBA activity only marginally reflected self-location ${ }^{59}$. More importantly, self-location, but not self-identification, has been shown to depend on the first-person perspective, as evidenced by studies in healthy subjects ${ }^{59}$ and in patients with heautoscopy and OBEs. As discussed above, this suggests that selflocation and the first-person perspective may rely on additional vestibular graviceptive (otolithic) signals and their integration with visual graviceptive and somatosensory signals and that this may require distinct brain processes.

I propose here that such distinct brain processes for self-location and the first-person perspective are localized in three regions within an area encompassing the posterior parietal cortex and the TPJ: the parietoinsular vestibular cortex (PIVC), area VIP and area MST (middle superior temporal) (FIG. 5b). In monkeys, these regions are densely packed with vestibular neurons, and many of these neurons also have large and bilateral visual receptive fields, as well as large and bilateral somatosensory receptive fields. The visual receptive fields are often $>50^{\circ}$, and somatosensory receptive fields respond to stimulation of neck, shoulder, trunk or even half or the entire body surface of the animal (see REFS 153-159 for the PIVC, REFS 90,158,159 for area VIP and REFS 158162 for area MST). In area VIP, for example, bimodal visuotactile neurons may encode the same visual and tactile movement direction ${ }^{89,90,104}$, and the receptive fields of some of these VIP neurons may cover the entire body and the entire visual field ${ }^{89}$ (FIG. 5b). As discussed above, some of these neurons may code for self-identification, but I speculate here that the subpopulation of VIP neurons that also respond to vestibular signals code for self-location and first-person perspective ${ }^{163,164}$ (FIG. 5b). Such trimodal neurons have been described in the TPJ regions PIVC ${ }^{153,154}$ and area MST ${ }^{165-168}$ in monkeys. MST neurons have further been implicated in the perception of heading direction based on the integration of visual and vestibular signals ${ }^{165,168}$. Accordingly, it could be argued that the neurologically and experimentally induced changes in the experienced direction of the first-person perspective and the associated changes in self-location are caused by absent or abnormal otolithic activity in visuovestibular neurons in area VIP, area MST and the PIVC. Otoliths are known to be ambiguous indicators of orientation in space and are activated in the same manner by gravitational and translational signals ${ }^{169}$; such ambiguity is often resolved by visual and somatosensory inputs. Accordingly, abnormal otolithic activity related to translation may be represented as a change in static orientation that is integrated with abnormal static visuotactile signals that indicate a static body position and an elevated and down-looking orientation. Although no direct evidence for this possibility yet exists, it could be speculated that in the supine position, trimodal PIVC, MST and VIP neurons may, during robotically induced down-looking, not only encode the position of the physical body but also a spatially distinct, elevated position that is inverted by $180^{\circ}$ and experienced as down-looking (FIG. 5c). The neuroimaging and neurological lesion data in humans reviewed above and the important role of the PIVC and area MST in visuovestibular processing together suggest that such otolithic vestibular and visuotactile mechanisms of the first-person perspective and self-location may occur in these two brain regions, whereas area VIP may encode all three aspects of bodily self-consciousness.

\section{Conclusions}

The 'I' of conscious experience is one of the most astonishing features of the human mind. Recent neuroscientific investigations of self-identification, self-location and first-person perspective have described some of the multisensory brain processes that may give rise to bodily self-consciousness. As argued elsewhere ${ }^{1,170}$, these three 

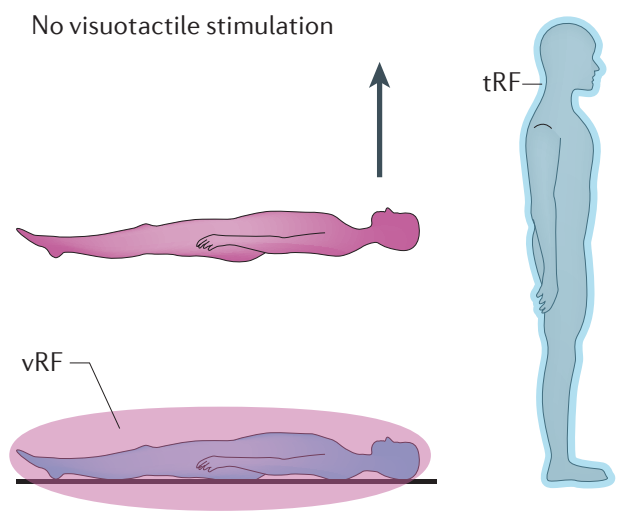

Synchronous visuotactile stimulation

b

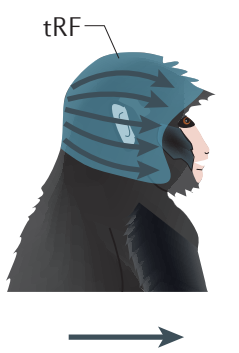

Vestibular stimulation

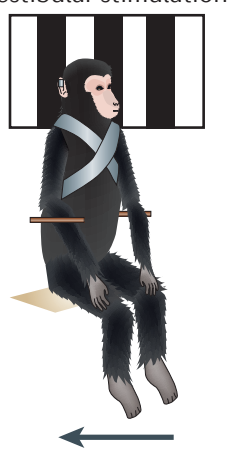

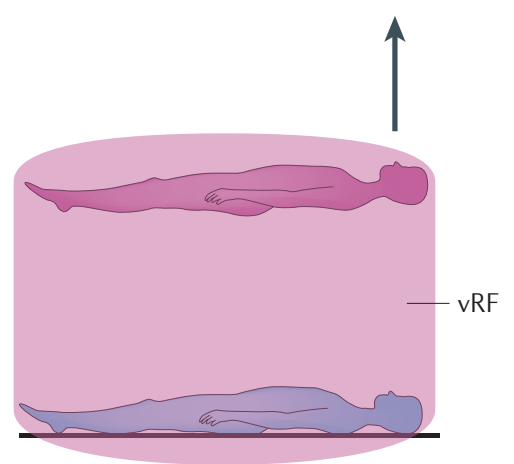

c

Synchronous visuotactile stimulation
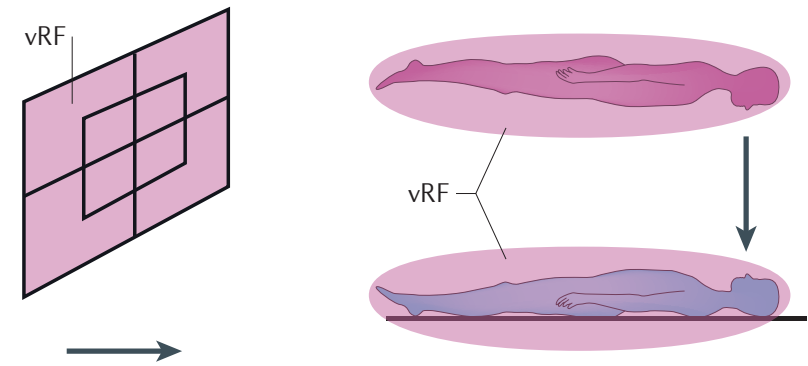

Figure 5 | Multimodal neurons in illusory self-location and the first-person perspective. a|Hypothetical changes in the size and/or position of the visual receptive field (vRF) of bimodal neurons in area VIP (ventral intraparietal) that may be associated with changes in self-location during the full-body illusion. The large vRF of a bimodal visuotactile neuron that responds to bilateral visual stimuli in the upper and lower visual field and in proximity to the subject's body is shown (pink area). The neuron's corresponding tactile RF (tRF) is shown in the middle panel and responds to touches applied to head, trunk, arms and legs (blue area). Seeing one's filmed body during the full-body illusion may alter the size and/or position of the vRFs (shown in pink) of such body-centred visuotactile neurons so that they extend to the more distant position of the seen filmed body (right panel), especially under conditions of synchronous visuotactile stroking. $\mathbf{b} \mid$ The receptive fields of a trimodal neuron in area VIP that responds to tactile, vestibular and visual signals is shown ${ }^{164}$. The left panel shows the location and size of the neuron's tRF (in blue), covering the entire head. The neuron is also direction-selective and encodes selectively back-to-front motion within the tRF (motion direction is indicated by the arrow below the panel). The same neuron also responds to vestibular stimulation and selectively encodes backward translation of the animal (middle panel; motion direction is indicated by the arrow below the panel). Finally, this neuron also responds to visual stimulation (receding optic flow and motion direction are indicated by the arrow below the panel). Similar neurons have been described in areas of the PIVC (parieto-insular vestibular cortex) $)^{153,154}$ and area MST (middle superior temporal) ${ }^{160-162}$.c | Hypothetical changes in the size and/or position of the vRF of trimodal neurons in area VIP, the PIVC and area MST that may be associated with changes in self-location and a down-looking first-person perspective during the full-body illusion. The large vRF of a trimodal visuotactile-vestibular neuron that responds to stimuli applied to bilateral visual stimuli in the upper and lower visual field and in proximity to the subject's body is shown (pink). Seeing a filmed body during the full-body illusion (induced by synchronous visuotactile stimulation) that was filmed in a posture and direction (upward) that is incongruent with the direction of veridical gravity (downward) may alter visuovestibular coding by trimodal neurons and induce abnormal otolithic perception. This association of abnormal otolithic signals that are integrated with abnormal visuotactile signals (during the full-body illusion) may lead to a change in perceived body orientation of the participant and may be associated with changes in the size and/or position of vRFs that also respond to the more distant position of the seen filmed body.

aspects are the necessary constituents of the simplest form of self-consciousness that arises when the brain encodes the origin of the first-person perspective from within a spatial frame of reference (that is, self-location) associated with self-identification. The reviewed data highlight the role of the PMC, IPS, EBA, sensorimotor cortex and - concerning self-location and the firstperson perspective - the temporoparietal cortex in bodily self-consciousness as informed by multisensory and vestibular signals. I propose that self-identification depends on somatosensory and visual signals and involves bimodal visuotactile neurons, and that selflocation and the first-person perspective depend on the integration of these bodily signals with vestibular cues in trimodal visuotactile-vestibular neurons. These differences between self-identification versus self-location and first-person perspective are corroborated by neuroimaging and neurological data; these data show that self-identification recruits primarily bilateral PMC and IPS and that self-location and first-person perspective 
recruit posterior parietal-TPJ regions (such as area MST and the PIVC) with a right hemispheric predominance. Posterior IPS regions such as area VIP may be involved in both self-identification and self-location. The contributions of other regions (such as the EBA and sensorimotor cortex) have been less robust across studies. Although much of the present Review may suggest that self-location and first-person perspective rely on the same brain mechanisms, neurological observations suggest that these aspects of bodily self-consciousness are in fact different and probably based on at least partially distinct brain mechanisms ${ }^{105}$.

The reviewed data extend other prominent proposals concerning the neural basis of bodily self-consciousness that have highlighted brain processes related to internal states of the body, such as interoceptive and homeostatic systems (for example, the heartbeat) as important signals, and that have highlighted the contribution of either the insula ${ }^{171}$ or the posterior medial parietal cortex ${ }^{172,173}$. Future research should explore the interactions between exteroceptive bodily signals (which the present Review focused on) and interoceptive and sensorimotor signals ${ }^{5,174}$, their relevance for self-consciousness and their distinct and common brain mechanisms ${ }^{175}$.

A cautionary note is warranted. The current amount of experimental data on bodily self-consciousness is very limited. Moreover, responses are often variable within and between subjects and are rarely based on repeated measures. Future research should include more precise measurements and more rigorous approaches based on psychophysical and, eventually, mathematical approaches. Additional research should be carried out, analysing functional and anatomical connectivity ${ }^{176,177}$ between the brain regions of bodily self-consciousness and how they relate to the so-called default mode network $^{176}$, which has been associated with self-related processing ${ }^{178}$. Further technological advances in virtual reality and robotics and their merging with cognitive science, brain imaging and clinical work are needed to provide the necessary bodily stimulations and the induction of stronger and prolonged altered states of self-consciousness. Comparable research needs to be carried out in non-human primates and rodents. These lines of research are likely to provide much ground for fascinating future research in neuroscience and experimental psychology as well as philosophy of mind and ethics.

\section{Future directions}

How does self-consciousness relate to the much larger field of consciousness studies? Empirical research into brain mechanisms of consciousness has mainly focused on behavioural and neural differences between conscious and unconscious processing of stimuli, mostly in the visual system ${ }^{179,180}$. Can such consciousness arise without or during strongly altered self-consciousness? This is an interesting topic for future research, and would require integrating studies of bodily self-consciousness with research into conscious and unconscious target detection, binocular rivalry, bistable perception, change blindness and other perceptions in well-defined psychophysical settings ${ }^{181}$. Bodily self-consciousness may turn out to be an important component for consciousness generally ${ }^{173}$. Certainly "it is not enough to say that the mind (and consciousness) is embodied. You also have to say how"182, and bodily self-consciousness may provide this link.

Cognitive psychologists and neuroscientists have studied many different aspects of the self (see REFS $2,4,5,125,129,183-190)$. Future work should address how self-consciousness is linked to language (for example, see REFS 117,191) and to memory and future prediction ${ }^{173}$ (also see REFS 192,193) to study 'narrative' and 'extended' aspects of self-consciousness, respectively. The behavioural and neural mechanisms of bodily self-consciousness should further be studied jointly with self-related processes such as perceptual and imagined viewpoint changes ${ }^{129}$, theory-of-mind and mentalizing ${ }^{194}$, empathy, and with consciousness in the motor and sensorimotor systems $^{5,195}$.

Finally, recent developments in virtual reality and robotics are opening novel avenues for human enhancement of sensorimotor and cognitive function. Virtual reality has already played an important part in augmenting cognition, assisting motor rehabilitation and as an effective treatment in anxiety and specific phobias ${ }^{196,197}$. These treatments may be complemented and improved by including automatized manipulations of illusory hand ownership, self-location and self-identification. Diagnosis and treatments of other medical conditions such as pain syndromes $^{76,198}$ and inflammation ${ }^{199}$ may also benefit from artificially induced changes in hand ownership, selflocation and self-identification. Finally, the manipulation of bodily self-consciousness may generate and enhance bodily feelings for de-afferented body parts in patients with tetra- and paraplegia. Recent work in patients with amputations - initiated by Ramachandran and colleagues $^{64}$ - has already explored these possibilities for the design of future artificial limbs ${ }^{200,201}$. Patients with severely disabling and chronic conditions, such as paraand tetraplegia following spinal cord injury, may profit in the future from self-enhanced or augmented artificial bodies, allowing them not only to move but also to feel the 'digital body' as their own body ${ }^{202-205}$.
1. Blanke, O. \& Metzinger, T. Full-body illusions and minimal phenomenal selfhood. Trends Cogn. Sci. 13, 7-13 (2009).

2. Christoff, K., Cosmelli, D., Legrand, D. \& Thompson, E. Specifying the self for cognitive neuroscience. Trends Cogn. Sci. 15, 104-112 (2011).

3. de Vignemont, F. Embodiment, ownership and disownership. Conscious. Cogn. 20, 82-93 (2011). Jeannerod, $M$. The mechanism of self-recognition in humans. Behav. Brain Res. 142, 1-15 (2003).

5. Knoblich, G. Self-recognition: body and action. Trends Cogn. Sci. 6, 447-449 (2002).
6. Legrand, D. Pre-reflective self-as-subject from experiential and empirical perspectives. Conscious. Cogn. 16, 583-599 (2007).

7. Berlucchi, G. \& Aglioti, S. The body in the brain: neural bases of corporeal awareness. Trends Neurosci. 20, 560-564 (1997).

A comprehensive, classical review of deficits in body perception that are of relevance for bodily self-consciousness following brain damage in patients with neurological disorders.

8. Berlucchi, G. $\&$ Aglioti, S. M. The body in the brain revisited. Exp. Brain Res. 200, 25-35 (2010).
9. Critchley, M. The body-image in neurology. Lancet 255, 335-341 (1950)

10. Head, H. \& Holmes, G. Sensory disturbances from cerebral lesions. Brain 34, 102-254 (1911).

11. Hécaen, H. \& Ajuriaguerra, J. (eds) Meconnassiances et Hallucinations Corporelles: Intégration et Désintégration de la Somatognosie 310-343 (Masson, 1952) (in French).

12. Lhermitte, J. L'image de Notre Corps 170-227 (L'Harmattan, 1998) (in French).

13. Schilder, P. The Image and Appearance of the Human Body (Georg Routledge and Sons, 1935). 
14. Sollier, P. Les Phénomènes d'Autoscopie (Alcan, 1903) (in French).

15. Gerstmann, J. Problem of imperception of disease and of impaired body territories with organic lesions. Arch. Neurol. Psychiatry 48, 890-913 (1942).

16. Halligan, P. W., Marshall, J. C. \& Wade, D. T. Unilateral somatoparaphrenia after right hemisphere stroke: a case description. Cortex 31, 173-182 (1995).

17. Fotopoulou, A. et al. Mirror-view reverses somatoparaphrenia: dissociation between first- and third-person perspectives on body ownership. Neuropsychologia 49, 3946-3955 (2011).

18. Vallar, G. \& Ronchi, R. Somatoparaphrenia: a body delusion. A review of the neuropsychological literature. Exp. Brain Res. 192, 533-551 (2009).

19. Baier, B. \& Karnath, H. O. Tight link between our sense of limb ownership and self-awareness of actions. Stroke 39, 486-488 (2008)

20. Botvinick, M. \& Cohen, J. Rubber hands 'feel' touch that eyes see. Nature 391, 756 (1998).

21. Ehrsson, H. H., Spence, C. \& Passingham, R. E. That's my hand! Activity in premotor cortex reflects feeling of ownership of a limb. Science 305, 875-877 (2004).

22. Slater, M., Perez-Marcos, D., Ehrsson, H. H. \& Sanchez-Vives, M. V. Towards a digital body: the virtual arm illusion. Front. Hum. Neurosci. 2, 6 (2008).

23. Tsakiris, M. \& Haggard, P. The rubber hand illusion revisited: visuotactile integration and self-attribution J. Exp. Psychol. Hum. Percept. Perform. 31, 80-91 (2005).

24. Kammers, M. P., de Vignemont, F., Verhagen, L. \& Dijkerman, H. C. The rubber hand illusion in action. Neuropsychologia 47, 204-211 (2009)

25. Lloyd, D. M. Spatial limits on referred touch to an alien limb may reflect boundaries of visuo-tactile peripersonal space surrounding the hand. Brain Cogn 64, 104-109 (2007)

26. Makin, T. R., Holmes, N. P. \& Ehrsson, H. H. On the other hand: dummy hands and peripersonal space. Behav. Brain Res. 191, 1-10 (2008).

27. Tsakiris, M. My body in the brain: a neurocognitive model of body-ownership. Neuropsychologia 48, 703-712 (2010)

28. Rohde, M., Di Luca, M. \& Ernst, M. O. The Rubber Hand Illusion: feeling of ownership and proprioceptive drift do not go hand in hand. PLOS ONE 6, e21659 (2011).

29. Ehrsson, H. H., Holmes, N. P. \& Passingham, R. E. Touching a rubber hand: feeling of body ownership is associated with activity in multisensory brain areas. J. Neurosci. 25, 10564-10573 (2005).

30. Kammers, M. P. et al. Is this hand for real? Attenuation of the rubber hand illusion by transcranial magnetic stimulation over the inferior parietal lobule. J. Cogn. Neurosci. 21, 1311-1320 (2009).

31. Kanayama, N., Sato, A. \& Ohira, H. Crossmodal effect with rubber hand illusion and gamma-band activity. Psychophysiology 44, 392-402 (2007)

32. Kanayama, N., Sato, A. \& Ohira, H. The role of gamma band oscillations and synchrony on rubber hand illusion and crossmodal integration. Brain Cogn. 69 19-29 (2009)

33. Tsakiris, M., Hesse, M. D., Boy, C., Haggard, P. \& Fink, G. R. Neural signatures of body ownership: a sensory network for bodily self-consciousness. Cereb. Cortex 17, 2235-2244 (2007)

34. Ehrsson, H. H., Wiech, K., Weiskopf, N., Dolan, R. J. \& Passingham, R. E. Threatening a rubber hand that you feel is yours elicits a cortical anxiety response. Proc. Natl Acad. Sci. USA 104, 9828-9833 (2007).

35. Lloyd, D., Morrison, I. \& Roberts, N. Role for human posterior parietal cortex in visual processing of aversive objects in peripersonal space. J. Neurophysiol 95, 205-214 (2006).

36. Zeller, D., Gross, C., Bartsch, A., Johansen-Berg, H. \& Classen, J. Ventral premotor cortex may be required for dynamic changes in the feeling of limb ownership: a lesion study. J. Neurosci. 31, 4852-4857 (2011)

37. Bremmer, F. et al. Polymodal motion processing in posterior parietal and premotor cortex: a human fMRI study strongly implies equivalencies between humans and monkeys. Neuron 29, 287-296 (2001)

38. Fogassi, L. et al. Coding of peripersonal space in inferior premotor cortex (area F4). J. Neurophysiol. 76, 141-157 (1996)

39. Gentile, G., Petkova, V. I. \& Ehrsson, H. H. Integration of visual and tactile signals from the hand in the human brain: an FMRI study. J. Neurophysiol. 105 910-922 (2011)

40. Graziano, M. S. \& Gandhi, S. Location of the polysensory zone in the precentral gyrus of anesthetized monkeys. Exp. Brain Res. 135, 259-266 (2000)

41. Graziano, M. S., Hu, X. T. \& Gross, C. G. Visuospatial properties of ventral premotor cortex. J. Neurophysiol. 77, 2268-2292 (1997).

42. Iriki, A., Tanaka, M. \& Iwamura, Y. Coding of modified body schema during tool use by macaque postcentral neurones. Neuroreport 7, 2325-2330 (1996).

43. Maravita, A. \& Iriki, A. Tools for the body (schema). Trends Cogn. Sci. 8, 79-86 (2004).

An important review on multisensory integration of the upper extremity, in particular the integration of visual, tactile and proprioceptive signals in the parietal cortex of human and non-human primates.

44. Petkova, V. I. et al. From part- to whole-body ownership in the multisensory brain. Curr. Biol. 21 , 1118-1122 (2011). An important study on the brain mechanisms of self-identification using fMRI and virtual reality.

45. Farne, A., Iriki, A. \& Ladavas, E. Shaping multisensory action-space with tools: evidence from patients with cross-modal extinction. Neuropsychologia 43 , 238-248 (2005)

46. Holmes, N. P., Calvert, G. A. \& Spence, C. Tool use changes multisensory interactions in seconds: evidence from the crossmodal congruency task. Exp. Brain Res. 183, 465-476 (2007).

47. Maravita, A., Spence, C., Sergent, C. \& Driver, J. Seeing your own touched hands in a mirror modulates cross-modal interactions. Psychol. Sci. 13, 350-355 (2002).

48. Graziano, M. S., Cooke, D. F. \& Taylor, C. S. Coding the location of the arm by sight. Science 290 . 1782-1786 (2000)

Important research in macaque monkeys on the neurophysiology of area 5 neurons and the integration of visual, proprioceptive and tactile cues. These findings are of relevance for self-attribution of an individual's hand.

49. Dieguez, S., Mercier, M. R., Newby, N. \& Blanke, O. Feeling numbness for someone else's finger. Curr. Biol. 19, R1108-R1109 (2009).

50. Tastevin, J. En partant de lexpérience d'Aristote: les déplacements artificiels des parties du corps ne sont pas suivis par le sentiment de ces parties ni pas les sensations qu'on peut y produire. Encephale 1 140-158 (1937) (in French).

51. Brugger, P., Regard, M. \& Landis, T. Unilaterally felt presences: the neuropsychiatry of one's invisible Doppelgänger. Neuropsychiatry Neuropsychol. Behav. Neurol. 9, 114-122 (1996).

52. Heydrich, L., Dieguez, S., Grunwald, T., Seeck, M. \& Blanke, O. Illusory own body perceptions: case reports and relevance for bodily self-consciousness. Conscious. Cogn. 19, 702-710 (2010).

53. Sforza, A., Bufalari, I., Haggard, P. \& Aglioti, S. M. My face in yours: visuo-tactile facial stimulation influences sense of identity. Soc. Neurosci. 5, 148-162 (2010).

54. Tsakiris, M. Looking for myself: current multisensory input alters self-face recognition. PLOS ONE 3, e4040 (2008)

55. Bolognini, N., Ladavas, E. \& Farne, A. Spatial perspective and coordinate systems in autoscopy: a case report of a "fantome de profil" in occipital brain damage. J. Cogn. Neurosci. 23, 1741-1751 (2011).

56. Devinsky, O., Feldmann, E., Burrowes, K. \& Bromfield, E. Autoscopic phenomena with seizures. Arch. Neurol. 46, 1080-1088 (1989)

57. Grusser, O. J. \& Landis, T. in Visual Agnosia and Other Disturbances of Visual Perception and Cognition (eds Grusser, O. J. \& Landis, T.) 297-303 (Macmillan, 1991).

8. Ehrsson, H. H. The experimental induction of out-of-body experiences. Science 317, 1048 (2007).

59. Ionta, S. et al. Multisensory mechanisms in temporoparietal cortex support self-location and first-person perspective. Neuron 70, 363-374 (2011). An important study on the brain mechanisms of self-identification, self-location and the first-person perspective using fMRI with neuroscience robotics.

60. Lenggenhager, B., Tadi, T., Metzinger, T. \& Blanke, O. Video ergo sum: manipulating bodily selfconsciousness. Science 317, 1096-1099 (2007).

61. Lenggenhager, B., Mouthon, M. \& Blanke, O. Spatial aspects of bodily self-consciousness. Conscious. Cogn. 18, 110-117 (2009)

62. Altschuler, E. L. \& Ramachandran, V. S. A simple method to stand outside oneself. Perception 36 632-634 (2007).

63. Mizumoto, M. \& Ishikawa, M. Immunity to error through misidentification and the bodily illusion experiment J. Conscious. Stud. 12, 3-19 (2005).
64. Ramachandran, V. S., Rogers-Ramachandran, D. \& Cobb, S. Touching the phantom limb. Nature 377 489-490 (1995)

65. Stratton, G. M. The spatial harmony of touch and sight. Mind 8 492-505 (1899).

66. von Helmholtz, H. Helmholtz's Treatise on Physiological Optics (Dover Publication, 1962).

67. Spence, C., Pavani, F. \& Driver, J. Spatial constraints on visual-tactile cross-modal distractor congruency effects. Cogn. Affect Behav. Neurosci. 4, 148-169 (2004).

68. Aspell, J. E., Lenggenhager, B. \& Blanke, O. Keeping in touch with one's self: multisensory mechanisms of selfconsciousness. PLoS ONE 4, e6488 (2009).

69. Igarashi, Y., Kimura, Y., Spence, C. \& Ichihara, S. The selective effect of the image of a hand on visuotactile interactions as assessed by performance on the crossmodal congruency task. Exp. Brain Res. 184, 31-38 (2008)

70. Pavani, F. \& Castiello, U. Binding personal and extrapersonal space through body shadows. Nature Neurosci. 7, 14-16 (2004).

71. Pavani, F., Spence, C. \& Driver, J. Visual capture of touch: out-of-the-body experiences with rubber gloves. Psychol. Sci. 11, 353-359 (2000).

72. Shore, D. I., Barnes, M. E. \& Spence, C. Temporal aspects of the visuotactile congruency effect. Neurosci. Lett. 392, 96-100 (2006).

73. Aspell, J. E., Lavanchy, T., Lenggenhager, B. \& Blanke, O. Seeing the body modulates audiotactile integration. Eur. J. Neurosci. 31, 1868-1873 (2010).

74. Zopf, R., Savage, G. \& Williams, M. A. Crossmodal congruency measures of lateral distance effects on the rubber hand illusion. Neuropsychologia 48, 713-725 (2010).

75. Petkova, V. I. \& Ehrsson, H. H. If I were you: perceptual illusion of body swapping. PLOS ONE 3, e3832 (2008)

76. Hansel, A., Lenggenhager, B., von Kanel, R., Curatolo, M. \& Blanke, O. Seeing and identifying with a virtual body decreases pain perception. Eur. J. Pain 15, 874-879 (2011)

77. Lenggenhager, B., Halje, P. \& Blanke, O. Alpha band oscillations correlate with illusory self-location induced by virtual reality. Eur. J. Neurosci. 33, 1935-1943 (2011).

78. Pineda, J. A. The functional significance of mu rhythms: translating "seeing" and "hearing" into “doing”. Brain Res. Brain Res. Rev. 50, 57-68 (2005).

79. Oakes, T. R. et al. Functional coupling of simultaneous electrical and metabolic activity in the human brain. Hum. Brain Mapp. 21, 257-270 (2004)

80. Gastaut, H. Etude électrocorticographique de la réactivité des rhythms rolandiques. Rev. Neurol. (Paris) 87, 176-182 (1952) (in French).

81. Pfurtscheller, G. \& Neuper, C. Motor imagery activates primary sensorimotor area in humans. Neurosci. Lett. 239, 65-68 (1997)

82. Ulloa, E. R. \& Pineda, J. A. Recognition of point-light biological motion: mu rhythms and mirror neuron activity. Behav. Brain Res. 183, 188-194 (2007).

83. Pfurtscheller, G. Central beta rhythm during sensorimotor activities in man. Electroencephalogr Clin. Neurophysiol. 51, 253-264 (1981).

84. Cheyne, D. et al. Neuromagnetic imaging of cortical oscillations accompanying tactile stimulation. Brain Res. Cogn. Brain Res. 17, 599-611 (2003).

85. Astafiev, S. V., Stanley, C. M., Shulman, G. L. \& Corbetta, M. Extrastriate body area in human occipital cortex responds to the performance of motor actions. Nature Neurosci. 7, 542-548 (2004).

86. Downing, P. E., Jiang, Y., Shuman, M. \& Kanwisher, N A cortical area selective for visual processing of the human body. Science 293, 2470-2473 (2001).

87. Grossman, E. D. \& Blake, R. Brain areas active during visual perception of biological motion. Neuron 35 , 1167-1175 (2002)

88. Urgesi, C., Candidi, M., Ionta, S. ¿ Aglioti, S. M. Representation of body identity and body actions in extrastriate body area and ventral premotor cortex. Nature Neurosci. 10, 30-31 (2007).

89. Duhamel, J. R., Colby, C. L. \& Goldberg, M. E. in Brain and Space (ed. Paillard, J.) 223-236 (Oxford Univ. Press, 1991).

90. Duhamel, J. R., Colby, C. L. \& Goldberg, M. E. Ventral intraparietal area of the macaque: congruent visual and somatic response properties. J. Neurophysiol. 79 126-136 (1998)

Pioneering work on the neurophysiology of VIP neurons and the integration of visual and tactile cues that are likely to be of relevance for self-identification. 
91. Armel, K. C. \& Ramachandran, V. S. Projecting sensations to external objects: evidence from skin conductance response. Proc. Biol. Sci. 270, 1499-1506 (2003).

92. Taoka, M., Toda, T., Iriki, A., Tanaka, M. \& Iwamura, Y. Bilateral receptive field neurons in the hindlimb region of the postcentral somatosensory cortex in awake macaque monkeys. Exp. Brain Res. 134, 139-146 (2000).

93. Taoka, M., Toda, T. \& Iwamura, Y. Representation of the midline trunk, bilateral arms, and shoulders in the monkey postcentral somatosensory cortex. Exp. Brain Res. 123, 315-322 (1998)

94. Sakata, H., Taira, M., Murata, A. \& Mine, S. Neural mechanisms of visual guidance of hand action in the parietal cortex of the monkey. Cereb. Cortex $\mathbf{5}$, 429-438 (1995).

95. Kitada, R., Johnsrude, I. S., Kochiyama, T. \& Lederman, S. J. Functional specialization and convergence in the occipito-temporal cortex supporting haptic and visual identification of human faces and body parts: an fMRI study. J. Cogn. Neurosci. 21, 2027-2045 (2009).

96. Cardini, F. et al. Viewing one's own face being touched modulates tactile perception: an fMRI study. J. Cogn. Neurosci. 23, 503-513 (2011).

97. Brugger, P. Reflective mirrors: perspective-taking in autoscopic phenomena. Cogn. Neuropsychiatry 7 , 179-194 (2002)

98. Blanke, O. \& Mohr, C. Out-of-body experience, heautoscopy, and autoscopic hallucination of neurological origin: implications for neurocognitive mechanisms of corporeal awareness and selfconsciousness. Brain Res. Brain Res. Rev. 50, 184-199 (2005)

A useful review about the neurological findings in a large number of patients suffering from autoscopic phenomena that are associated with abnormal bodily self-consciousness.

99. Lukianowicz, N. Autoscopic phenomena. AMA Arch Neurol. Psychiatry 80, 199-220 (1958).

100. Blanke, O., Landis, T., Spinelli, L. \& Seeck, M Out-of-body experience and autoscopy of neurological origin. Brain 127, 243-258 (2004).

101. Brugger, P., Agosti, R., Regard, M., Wieser, H. G. \& Landis, T. Heautoscopy, epilepsy, and suicide. J. Neurol. Neurosurg. Psychiatry 57, 838-839 (1994).

102. Pearson, J. \& Dewhurst, K. Two cases of heautoscopic phenomena following organic lesions. Encephale 43. 166-172 (1954)

103. Lunn, V. Autoscopic phenomena. Acta Psych Scand. 46, 118-125 (1970)

104. Avillac, M., Deneve, S., Olivier, E., Pouget, A. \& Duhamel, J. R. Reference frames for representing visual and tactile locations in parietal cortex. Nature Neurosci. 8, 941-949 (2005)

105. De Ridder, D., Van Laere, K., Dupont, P., Menovsky, T. $\varangle$ Van de Heyning, P. Visualizing out-of-body experience in the brain. N. Engl. J. Med. 357 1829-1833 (2007).

106. Claparede, E. D. Note sur la localisation du moi. Arch. Psychol. 19, 172-182 (1924) (in French).

107. Hoffmann, F. R. Über die Sehrichtungen. Graefe's Arch. Clin. Exp. Opthalmol. 116, 135-142 (1926) (in German)

108. Bertossa, F., Besa, M., Ferrari, R. \& Ferri, F. Point zero: a phenomenological inquiry into the seat of consciousness. Percept. Mot. Skills 107, 323-335 (2008).

109. Limanowski, J. \& Hecht, H. Where do we stand on locating the self? Psychology 2, 312-317 (2011)

110. Roelofs, C. O. Considerations on the visual egocenter. Acta Pschol. 16, 226-234 (1959).

111. Harris, C. S. Perceptual adaptation to inverted, reversed, and displaced vision. Psychol. Rev. 72, 419-444 (1965)

112. Held, R. \& Freedman, S. J. Plasticity in human sensorimotor control. Science 142, 455-462 (1963).

113. Kohler, I. Uber Aufbau und Wandlungen der Wahrnehmungswelt. Östereichische Akademie der Wissenschaften. Philosophisch historische Klasse 227, 1-118 (1951) (in German).

114. Pisella, L., Rode, G., Farne, A., Tilikete, C. \& Rossetti, Y. Prism adaptation in the rehabilitation of patients with visuo-spatial cognitive disorders. Curr. Opin. Neurol. 19, 534-542 (2006).

115. Welch, R. B. Research on adaptation to rearranged vision: 1966-1974. Perception 3, 367-392 (1974).
116. Brugger, P., Regard, M. \& Landis, T. Illusory reduplication of one's own body: phenomenology and classification of autoscopic phenomena. Cogn. Neuropsychiatry 2, 19-38 (1997).

117. Dennett, D. C. Consciousness Explained (Penguin Books, 1991).

118. Nagel, T. The View from Nowhere (Oxford Univ. Press, 1986).

119. Shoemaker, S. The First-person Perspective and Other Essays (Cambridge Univ. Press, 1996).

120. Blanke, O., Ortigue, S., Landis, T. \& Seeck, M Stimulating illusory own-body perceptions. Nature 419, 269-270 (2002)

121. Penfield, W. \& Jaspers, H. Epilepsy and the Functional Anatomy of the Human Brain (Little Brown \& Co, 1954).

122. Tong, F. Out-of-body experiences: from Penfield to present. Trends Cogn. Sci. 7, 104-106 (2003).

123. Brandt, C., Brechtelsbauer, D., Bien, C. G. \& Reiners, K. Out-of-body experience as possible seizure symptom in a patient with a right parietal lesion]. Nervenarzt 76, 1259-1262 (2005) (in German)

124. Maillard, L., Vignal, J. P., Anxionnat, R. \& TaillandierVespignani, L. Semiologic value of ictal autoscopy. Epilepsia 45, 391-394 (2004).

125. Arzy, S., Thut, G., Mohr, C., Michel, C. M. \& Blanke, O. Neural basis of embodiment: distinct contributions of temporoparietal junction and extrastriate body area. J. Neurosci. 26, 8074-8081 (2006)

126. Petkova, V. I., Khoshnevis, M. \& Ehrsson, H. H. The perspective matters! Multisensory integration in egocentric reference frames determines full-body ownership. Front. Psychol. 2, 35 (2011).

127. Slater, M., Spanlang, B., Sanchez-Vives, M. V. \& Blanke, O. First person experience of body transfer in virtual reality. PLOS ONE 5, e10564 (2010).

128. David, N. et al. Neural representations of self versus other: visual-spatial perspective taking and agency in a virtual ball-tossing game. J. Cogn. Neurosci. 18 898-910 (2006).

129. Vogeley, K. \& Fink, G. R. Neural correlates of the firstperson-perspective. Trends Cogn. Sci. 7, 38-42 (2003).

130. Vogeley, K. et al. Neural correlates of first-person perspective as one constituent of human selfconsciousness. J. Cogn. Neurosci. 16, 817-827 (2004).

131. Corradi-Dell'acqua, C. et al. Effects of shifting perspective of the self: an fMRI study. Neuroimage $\mathbf{4 0}$ 1902-1911 (2008)

132. Zacks, J. M. \& Michelon, P. Transformations of visuospatial images. Behav. Cogn. Neurosci. Rev. 4 , 96-118 (2005)

133. Burgess, N., Maguire, E. A., Spiers, H. J. \& O’Keefe, J. A temporoparietal and prefrontal network for retrieving the spatial context of lifelike events. Neuroimage 14, 439-453 (2001)

134. Lambrey, S. et al. Distinct visual perspective-taking strategies involve the left and right medial temporal lobe structures differently. Brain 131, 523-534 (2008)

135. Golomer, E., Cremieux, J., Dupui, P., Isableu, B. \& Ohlmann, T. Visual contribution to self-induced body sway frequencies and visual perception of male professional dancers. Neurosci. Lett. 267, 189-192 (1999)

136. Isableu, B., Ohlmann, T., Cremieux, J. \& Amblard, B. Selection of spatial frame of reference and postura control variability. Exp. Brain Res. 114, 584-589 (1997)

137. Lopez, C., Lacour, M., Magnan, J. \& Borel, L. Visual field dependence-independence before and after unilateral vestibular loss. Neuroreport 17, 797-803 (2006)

138. Young, L. R., Oman, C. M., Watt, D. G., Money, K. E. \& Lichtenberg, B. K. Spatial orientation in weightlessness and readaptation to earth's gravity. Science 225, 205-208 (1984).

139. Green, C. E. Out-of-Body Experiences (Hamish Hamilton, 1968)

140. Gurovskiy, N. N., Bryanov, I. I. \& Yegorov, A. D. Changes in the vestibular function during space flight. Acta Astronaut. 2, 207-216 (1975).

141. Kornilova, L. N. Orientation illusions in spaceflight. J. Vestib. Res. 7, 429-439 (1997)

142. Lackner, J. R. Spatial orientation in weightless environments. Perception 21, 803-812 (1992)

143. Graybiel, A. \& Kellogg, R. S. Inversion illusion in parabolic flight: its probable dependence on otolith function. Aerosp. Med. 38, 1099-1103 (1967).

144. Aubert, H. Eine scheinbare bedeutende Drehung von Objecten bei Neigung des Kopfes nach rechts oder links. Virchows Archiv. 20, 381-393 (1861) (in German).
145. Jenkin, H. L., Dyde, R. T., Jenkin, M. R., Howard, I. P. ¿ Harris, L. R. Relative role of visual and non-visual cues in determining the direction of "up": experiments in the York tilted room facility. J. Vestib. Res. 13, 287-293 (2003)

146. Lopez, C., Bachofner, C., Mercier, M. \& Blanke, O. Gravity and observer's body orientation influence the visual perception of human body postures. J. Vis. 9, 11-14 (2009).

147. Mittelstaedt, $\mathrm{H}$. The role of the otoliths in perception of the orientation of self and world to the vertical. Zool. Jahrb. Abt Physiol. 95, 418-425 (1991).

148. Lopez, C., Halje, P. \& Blanke, O. Body ownership and embodiment: vestibular and multisensory mechanisms. Neurophysiol. Clin. 38, 149-161 (2008).

149. Solms, M., Kaplan-Solms, K., Saling, M. \& Miller, P. Inverted vision after frontal lobe disease. Cortex $\mathbf{2 4}$ 499-509 (1988)

150. Tiliket, C., Ventre-Dominey, J., Vighetto, A. \& Grochowicki, M. Room tilt illusion. A central otolith dysfunction. Arch. Neurol. 53, 1259-1264 (1996).

151. Chan, A. W., Peelen, M. V. \& Downing, P. E. The effect of viewpoint on body representation in the extrastriate body area. Neuroreport 15, 2407-2410 (2004).

152. Saxe, R., Jamal, N. \& Powell, L. My body or yours? The effect of visual perspective on cortical body representations. Cereb. Cortex 16, 178-182 (2006).

153. Grusser, O. J., Pause, M. \& Schreiter, U. Vestibular neurones in the parieto-insular cortex of monkeys (Macaca fascicularis): visual and neck receptor responses. J. Physiol. 430, 559-583 (1990).

154. Grusser, O. J., Pause, M. \& Schreiter, U. Localization and responses of neurones in the parieto-insular vestibular cortex of awake monkeys (Macaca fascicularis). J. Physiol. 430, 537-557 (1990). Pioneering work on the neurophysiology of PIVC neurons and the integration of visual, vestibular and somatosensory cues that are likely to be of relevance for self-location and the first-person perspective.

155. Robinson, C. J. \& Burton, H. Somatotopographic organization in the second somatosensory area of M. fascicularis. J. Comp. Neurol. 192, 43-67 (1980)

156. Schneider, R. J., Friedman, D. P. \& Mishkin, M. A modality-specific somatosensory area within the insula of the rhesus monkey. Brain Res. 621 116-120 (1993)

157. Guldin, W. O., Akbarian, S. \& Grusser, O. J. Corticocortical connections and cytoarchitectonics of the primate vestibular cortex: a study in squirrel monkeys (Saimiri sciureus). J. Comp. Neurol. 326, 375-401 (1992).

158. Guldin, W. O. \& Grusser, O. J. Is there a vestibular cortex? Trends Neurosci. 21, 254-259 (1998).

159. Lopez, C. \& Blanke, O. The thalamocortical vestibular system in animals and humans. Brain Res. Rev. 67, 119-146 (2011).

A comprehensive review about the vestibular cortex and the processing of vestibular, visual and somatosensory signals including neurophysiological, neuroanatomical and neuroimaging data in animals and humans.

160. Duffy, C. J. MST neurons respond to optic flow and translational movement. J. Neurophysiol. $\mathbf{8 0}$ 1816-1827 (1998).

161. Duffy, C. J. \& Wurtz, R. H. Sensitivity of MST neurons to optic flow stimuli. I. A continuum of response selectivity to large-field stimuli. J. Neurophysiol. 65 1329-1345 (1991).

162. Tanaka, K \& Saito, H. Analysis of motion of the visual field by direction, expansion/contraction, and rotation cells clustered in the dorsal part of the medial superio temporal area of the macaque monkey. J. Neurophysiol. 62, 626-641 (1989).

163. Schlack, A., Hoffmann, K. P. \& Bremmer, F. Interaction of linear vestibular and visual stimulation in the macaque ventral intraparietal area (VIP). Eur. J. Neurosci. 16, 1877-1886 (2002).

164. Bremmer, F., Klam, F., Duhamel, J. R., Ben Hamed, S \& Graf, W. Visual-vestibular interactive responses in the macaque ventral intraparietal area (VIP). Eur J. Neurosci. 16, 1569-1586 (2002). Important work on the neurophysiology of VIP neurons and the integration of visual, vestibular and somatosensory cues that are likely to be of relevance for self-identification, self-location and the first-person perspective.

165. Bremmer, F., Kubischik, M., Pekel, M., Lappe, M. \& Hoffmann, K. P. Linear vestibular self-motion signals in monkey medial superior temporal area. Ann. NY Acad. Sci. 871, 272-281 (1999). 
166. Fetsch, C. R., Wang, S., Gu, Y., Deangelis, G. C. \& Angelaki, D. E. Spatial reference frames of visual, vestibular, and multimodal heading signals in the dorsal subdivision of the medial superior temporal area. J. Neurosci. 27, 700-712 (2007).

167. Gu, Y., Angelaki, D. E. \& Deangelis, G. C. Neural correlates of multisensory cue integration in macaque MSTd. Nature Neurosci. 11, 1201-1210 (2008).

168. Gu, Y., DeAngelis, G. C. \& Angelaki, D. E. A functional link between area MSTd and heading perception based on vestibular signals. Nature Neurosci. 10 1038-1047 (2007).

169. MacNeilage, P. R., Banks, M. S., Berger, D. R. \& Bulthoff, H. H. A. Bayesian model of the disambiguation of gravitoinertial force by visual cues. Exp. Brain Res. 179, 263-290 (2007).

170. Metzinger, T. Being No One (MIT Press, 2003).

171. Craig, A. D. How do you feel - now? The anterior insula and human awareness. Nature Rev. Neurosci. 10, 59-70 (2009)

172. Damasio, A. \& Meyer, D. E. in The Neurology of Consciousness (eds Laureys, S. \& Tononi, G.) 3-14 (Elsevier, 2009).

173. Damasio, A. R. The Feeling of What Happens: Body and Emotion in the Making of Consciousness (Harcourt Brace, 1999).

174. Pacherie, E. The phenomenology of action: a conceptual framework. Cognition 107, 179-217 (2008).

175. Tsakiris, M., Tajadura-Jimenez, A. \& Costantini, M. Just a heartbeat away from one's body: interoceptive sensitivity predicts malleability of body-representations Proc. Biol. Sci. 278, 2470-2476 (2011)

176. Fox, M. D. \& Raichle, M. E. Spontaneous fluctuations in brain activity observed with functional magnetic resonance imaging. Nature Rev. Neurosci. 8 , 700-711 (2007)

177. Greicius, M. D., Supekar, K., Menon, V. \& Dougherty, R. F. Resting-state functional connectivity reflects structural connectivity in the default mode network. Cereb. Cortex 19, 72-78 (2009).

178. Golland, Y. et al. Extrinsic and intrinsic systems in the posterior cortex of the human brain revealed during natural sensory stimulation. Cereb. Cortex 17, 766-777 (2007)

179. Crick, F. \& Koch, C. Some reflections on visual awareness. Cold Spring Harb. Symp. Quant. Biol. 55 953-962 (1990)

180. Weiskrantz, L. Consciousness Lost and Found (Oxford Univ. Press, 1997)

181. Dehaene, S. \& Changeux, J. P. Experimental and theoretical approaches to conscious processing. Neuron 70, 200-227 (2011).

182. Edelman, G. Bright Air, Brilliant Fire (Basic Books, 1992).

183. Esslen, M., Metzler, S., Pascual-Marqui, R. \& Jancke, L. Pre-reflective and reflective self-reference: a spatiotemporal EEG analysis. Neuroimage 42 437-449 (2008)

184. Gillihan, S. J. \& Farah, M. J. Is self special? A critical review of evidence from experimental psychology and cognitive neuroscience. Psychol. Bull. 131, 76-97 (2005).
185. Heatherton, T. F. et al. Medial prefrontal activity differentiates self from close others. Soc. Cogn. Affect Neurosci. 1, 18-25 (2006)

186. Legrand, D. \& Ruby, P. What is self-specific? Theoretical investigation and critical review of neuroimaging results. Psychol. Rev. 116, 252-282 (2009).

187. Macrae, C. N., Moran, J. M., Heatherton, T. F. Banfield, J. F. \& Kelley, W. M. Medial prefrontal activity predicts memory for self. Cereb. Cortex 14 647-654 (2004).

188. Northoff, G. et al. Self-referential processing in our brain - a meta-analysis of imaging studies on the self. Neuroimage 31, 440-457 (2006).

189. Perrin, F. et al. Neural mechanisms involved in the detection of our first name: a combined ERPs and PET study. Neuropsychologia 43, 12-19 (2005).

190. Platek, S. M. et al. Neural substrates for functionally discriminating self-face from personally familiar faces. Hum. Brain Mapp. 27, 91-98 (2006).

191. Gazzaniga, M. S., LeDoux, J. E. \& Wilson, D. H. Language, praxis, and the right hemisphere: clues to some mechanisms of consciousness. Neurology 27, 1144-1147 (1977).

192. Arzy, S., Arzouan, Y., Adi-Japha, E., Solomon, S. \& Blanke, O. The 'intrinsic' system in the human cortex and self-projection: a data driven analysis. Neuroreport 21, 569-574 (2010).

193. Arzy, S., Bick, A. \& Blanke, O. Mental time in amnesia: evidence from bilateral medial temporal damage before and after recovery. Cogn. Neuropsychol. 26, 503-510 (2009).

194. Saxe, R., Moran, J. M., Scholz, J. \& Gabrieli, J. Overlapping and non-overlapping brain regions for theory of mind and self reflection in individual subjects. Soc. Cogn. Affect Neurosci. 1, 229-234 (2006)

195. Moore, J. W., Lagnado, D., Deal, D. C. \& Haggard, P. Feelings of control: contingency determines experience of action. Cognition 110, 279-283 (2009)

196. Herbelin, B. Virtual Reality Exposure Therapy for Social Phobia. Thesis, Ecole Polytechnique Federale de Lausanne (2005).

197. Klinger, E. et al. Virtual reality therapy versus cognitive behavior therapy for social phobia: a preliminary controlled study. Cyberpsychol. Behav. 8, 76-88 (2005).

198. Moseley, G. L., Gallace, A. \& Spence, C. Space-based, but not arm-based, shift in tactile processing in complex regional pain syndrome and its relationship to cooling of the affected limb. Brain 132 , 3142-3151 (2009)

199. Barnsley, N. et al. The rubber hand illusion increases histamine reactivity in the real arm. Curr. Biol. 21, R945-R946 (2011)

200. Ehrsson, H. H. et al. Upper limb amputees can be induced to experience a rubber hand as their own. Brain 131, 3443-3452 (2008)

201. Marasco, P. D., Kim, K., Colgate, J. E., Peshkin, M. A $\&$ Kuiken, T. A. Robotic touch shifts perception of embodiment to a prosthesis in targeted reinnervation amputees. Brain 134, 747-758 (2011).
202. Blanke, O. \& Aspell, J. E. Brain technologies raise unprecedented ethical challenges. Nature 458, 703 (2009).

203. Hochberg, L. R. et al. Neuronal ensemble control of prosthetic devices by a human with tetraplegia. Nature 442, 164-171 (2006).

204. Nicolelis, M. Beyond Boundaries. The Neuroscience of Connecting Brains with Machines and How It will Change our Lives (Times Books, 2011).

205. Perez-Marcos, D., Slater, M. \& Sanchez-Vives, M. V. Inducing a virtual hand ownership illusion through a brain-computer interface. Neuroreport 20, 589-594 (2009).

206. Creem, S. H. et al. An fMRI study of imagined selfrotation. Cogn. Affect Behav. Neurosci. 1, 239-249 (2001).

207. Wraga, M., Shephard, J. M., Church, J. A., Inati, S. \& Kosslyn, S. M. Imagined rotations of self versus objects: an fMRI study. Neuropsychologia 43. 1351-1361 (2005)

208. Aichhorn, M., Perner, J., Kronbichler, M., Staffen, W. $\Sigma$ Ladurner, G. Do visual perspective tasks need theory of mind? Neuroimage 30, 1059-1068 (2006).

209. Zacks, J., Rypma, B. Gabrieli, J. D., Tversky, B. \& Glover, G. H. Imagined transformations of bodies: an fMRI investigation. Neuropsychologia 37 , 1029-1040 (1999)

210. Schwabe, L., Lenggenhager, B. \& Blanke, O. The timing of temporoparietal and frontal activations during mental own body transformations from different visuospatial perspectives. Hum. Brain Mapp. 30, 1801-1812 (2009).

211. Amorim, M. A. \& Stucchi, N. Viewer- and objectcentered mental explorations of an imagined environment are not equivalent. Brain Res. Cogn. Brain Res. 5, 229-239 (1997)

212. Wang, R. F. \& Simons, D. J. Active and passive scene recognition across views. Cognition 70, 191-210 (1999).

213. Clement, G., Moore, S. T., Raphan, T. \& Cohen, B. Perception of tilt (somatogravic illusion) in response to sustained linear acceleration during space flight. Exp. Brain Res. 138, 410-418 (2001).

214. Iriki, A., Tanaka, M., Obayashi, S. \& Iwamura, Y. Selfimages in the video monitor coded by monkey intraparietal neurons. Neurosci. Res. 40, 163-173 (2001).

\section{Acknowledgements}

The author thanks C. Pfeiffer for his valuable help on the manuscript. The author is supported by grants from the Swiss National Science Foundation (SINERGIA CRSII 1-125135), the European Science Foundation (FP7 project VERE) and the Bertarelli Foundation.

Competing interests statement

The author declares no competing financial interests.

FURTHER INFORMATION

Olaf Blanke's homepage: http://cnp.epfl.ch

ALL LINKS ARE ACTIVE IN THE ONLINE PDF 